\title{
The physics of snow crystals
}

\author{
Kenneth G Libbrecht
}

Norman Bridge Laboratory of Physics, California Institute of Technology 264-33, Pasadena, CA 91125, USA

E-mail: kgl@caltech.edu

Received 16 December 2004, in final form 10 January 2005

Published 8 March 2005

Online at stacks.iop.org/RoPP/68/855

\begin{abstract}
We examine the physical mechanisms governing the formation of snow crystals, treating this problem as a case study of the dynamics of crystal growth from the vapour phase. Particular attention is given to the basic theoretical underpinnings of the subject, especially the interplay of particle diffusion, heat diffusion and surface attachment kinetics during crystal growth, as well as growth instabilities that have important effects on snow crystal development.

The first part of this review focuses on understanding the dramatic variations seen in snow crystal morphology as a function of temperature, a mystery that has remained largely unsolved since its discovery 75 years ago. To this end we examine the growth of simple hexagonal ice prisms in considerable detail, comparing crystal growth theory with laboratory measurements of growth rates under a broad range of conditions. This turns out to be a surprisingly rich problem, which ultimately originates from the unusual molecular structure of the ice surface and its sensitive dependence on temperature. With new clues from precision measurements of attachment kinetics, we are now just beginning to understand these structural changes at the ice surface and how they affect the crystal growth process. We also touch upon the mostly unexplored topic of how dilute chemical impurities can greatly alter the growth of snow crystals.

The second part of this review examines pattern formation in snow crystals, with special emphasis on the growth of snow crystal dendrites. Again we treat this as a case study of the more general problem of dendritic growth during diffusion-limited solidification. Since snow crystals grow from the vapour, we can apply dendrite theory in the simplified slowgrowth limit where attachment kinetics dominates over capillarity in selecting the tip velocity. Although faceting is quite pronounced in these structures, many aspects of the formation of snow crystal dendrites are fairly well described using a theoretical treatment that does not explicitly incorporate faceting. We also describe electrically modified ice dendrite growth, which produces some novel needle-like structures.
\end{abstract}




\section{Contents}

1. Introduction 857

1.1. Ice as a case study $\quad 857$

1.2. Natural snow crystals $\quad 858$

1.3. The morphology diagram $\quad 858$

1.4. Complexity and symmetry 861

1.5. The physics of snow crystals $\quad 862$

2. Prism growth from vapour-theory $\quad 862$

2.1. Basic terminology $\quad 863$

2.2. Diffusion-limited growth 864

2.3. Diffusion modelling of prism growth 868

2.4. Facet growth with diffusion $\quad 869$

2.5. Attachment kinetics 870

$\begin{array}{ll}\text { 2.6. Surface structure and crystal growth } & 871\end{array}$

2.7. Chemically enhanced growth 874

3. Prism growth from vapour-observations $\quad 875$

3.1. Experimental techniques 875

3.2. Conclusions from growth measurements $\quad 878$

3.3. Summary of snow crystal prism growth $\quad 881$

4. Dendritic growth $\quad 882$

4.1. The transition from faceting to branching 882

4.2. Dendrite theory and snow crystal growth 884

$\begin{array}{ll}\text { 4.3. Full modelling of dendrite growth } & 887\end{array}$

4.4. Electrically and chemically modified ice dendrite growth 888

5. Outlook $\quad 890$

References $\quad 891$ 


\section{Introduction}

Snow crystals, also called snowflakes, are single crystals of ice that grow from water vapour. They form in copious numbers in the atmosphere and are well known for their elaborate, symmetrical patterns. The physics of snow crystal formation is a specific example of the more general problem of how crystals grow and develop, creating complex structures on many length scales in the process. Because crystallization is a basic phase transition and crystals make up the foundation of several major industries, much effort has been expended toward developing a detailed understanding of the physics of crystal nucleation and growth. There is also a considerable literature on pattern formation during solidification. Beyond the intrinsic scientific merit of these problems, the burgeoning commercial interest in the self-assembly of nano-scale devices has reinvigorated our desire to understand just how solidification produces ordered, and sometimes complex, structures from disordered precursors.

Structure formation during crystal growth is a rich many-body problem, for which there are few overarching theories and perhaps even fewer uncomplicated experimental systems. In part this reflects the fact that many factors contribute to crystal growth, including both largescale phenomena (e.g. particle and heat transport) and microscopic dynamics (e.g. surface diffusion and chemistry). Different crystals grown under different conditions exhibit a broad range of morphologies, growth rates and other characteristics, reflecting the variety of physical mechanisms that influence crystal growth. The inherent complexity of the physics has resulted in a rather large gap between the basic tenants of crystal growth theory and the phenomenology of growing practical crystals.

\subsection{Ice as a case study}

In many ways, the formation of ice crystals from the vapour phase is an excellent case study of crystal growth dynamics and pattern formation during solidification. Although it appears to be a relatively simple monomolecular physical system, the growth of snow crystals exhibits a surprisingly rich behaviour as a function of temperature, supersaturation and other external parameters. As we will see below, a great deal of this behaviour remains unexplained, even at a qualitative level. Thus there is much to be learned, and ample potential that a better understanding of ice will contribute to our overall understanding of crystal growth and solidification. Taking the next step beyond the monomolecular system, ice crystal growth from water vapour is known to be quite sensitive to chemical influences at the growing surface, so again ice is an excellent case study for the more general, and exceptionally diverse, problem of chemically mediated crystal growth.

While the physics of snow crystal formation is an excellent crystal growth problem, it also touches on several environmental and meteorological issues, simply because ice crystals often play major roles in atmospheric phenomena. For example, ice crystals are important in cloud electrification and lightning, via charging mechanisms that involve collisions between ice particles. These mechanisms depend on details of the ice surface structure that are still not well understood [1-3]. Also, chemical processes in the upper atmosphere frequently require the surfaces of ice crystals to boost their reaction rates $[4,5]$. Most meteorological phenomena involving ice particles, like the growth of snow crystals, are influenced to some degree by the structure and dynamics of the ice surface. Thus we expect they will share some common physics at a fundamental level. A better picture of the dynamics of the ice crystal surface during growth may also shed light on some of the many remaining mysteries surrounding the dynamics of the different solid and liquid states of water [6]. 


\subsection{Natural snow crystals}

Since snow crystals fall from the sky in symmetrical, patterned forms, they have been the source of considerable curiosity and scientific study for centuries (for detailed historical accounts see [7-11]). Johannes Kepler was apparently the first person to look at snow crystals with a scientific eye, writing a short treatise devoted to the subject in 1611, in which he describes the possible origins of snow crystal symmetry [12]. René Descartes penned an account of many different forms of natural snow crystals in 1637 as part of his famous treatise on weather phenomena, Les Météores (see [7]). These early investigations were followed by numerous accounts that described, in words and with sketches, the great variety of snow crystal morphologies found in natural snowfalls $[9,10,13,14]$. With the development of photography in the late 19th century, Wilson Bentley catalogued several thousand snow crystal images in 1931 that he had acquired over several decades [15]. Bentley's images popularized the snow crystal as a winter icon and were largely responsible for the widespread notion that no two snowflakes are alike.

Some examples of natural snow crystals are shown in figure 1. These are particularly well-formed and symmetrical specimens, chosen for their photographic appeal and as prime examples of several of the more common and/or interesting snow crystal forms. Natural snow crystals have been categorized into morphological types by several different authors $[9,17,18]$, acquiring names like stellar dendrites, spatial dendrites, sectored plates, hollow columns, bullet rosettes, capped columns and others [19]. Most natural snow crystals are smaller and less pristine than those shown in figure 1, and are generally more blocky in appearance, usually without the high degree of symmetry present in well-formed specimens. Polycrystalline forms are also exceedingly common. For an unbiased sample the reader is invited to go outside with a magnifying glass or microscope during a light snowfall. Colder snowfalls usually provide more symmetrical and faceted crystals, but even warmer conditions often bring some examples like those shown in figure 1.

\subsection{The morphology diagram}

The variety of snow crystal forms seen in Bentley's photographs prompted physicist Ukichiro Nakaya to perform the first in-depth laboratory study of snow crystal growth in the 1930s [9]. Nakaya not only categorized natural snow crystals under different meteorological conditions, he was also the first to grow synthetic snow crystals in the laboratory in a controlled environment. Nakaya observed the different growth morphologies that appeared at different temperatures and supersaturations and combined these observations into what is called a snow crystal morphology diagram, shown in figure 2. This diagram refers to snow crystals growing in air at a pressure near $1 \mathrm{~atm}$, so applies to natural snow crystals. Nakaya's results have been confirmed and supplanted by several subsequent investigations $[10,17,20]$, and the data have recently been extended to temperatures as low as $T=-70^{\circ} \mathrm{C}$ [21].

These observations reveal a surprisingly complex dependence of crystal morphology on temperature and supersaturation. Just below freezing, at temperatures near $T=-2^{\circ} \mathrm{C}$, the growth is plate-like, with thick plates at lower supersaturations, thinner plates at intermediate supersaturations, and plate-like dendritic structures at high supersaturations. For temperatures near $T=-5^{\circ} \mathrm{C}$, the growth is columnar, with stout columns at the lower supersaturations, more slender, often hollow columns at intermediate supersaturations, and clusters of thin, needle-like crystals at higher supersaturations. Colder still, near $T=-15^{\circ} \mathrm{C}$, the growth again becomes plate-like, and again one sees increasing structure with increasing supersaturation. The thin, plate-like crystals shown in figures $1(a)-(c)$ all grew at temperatures near $T=-15^{\circ} \mathrm{C}$. Finally, at the lowest temperatures the growth becomes a mixture of thick plates at low supersaturations 

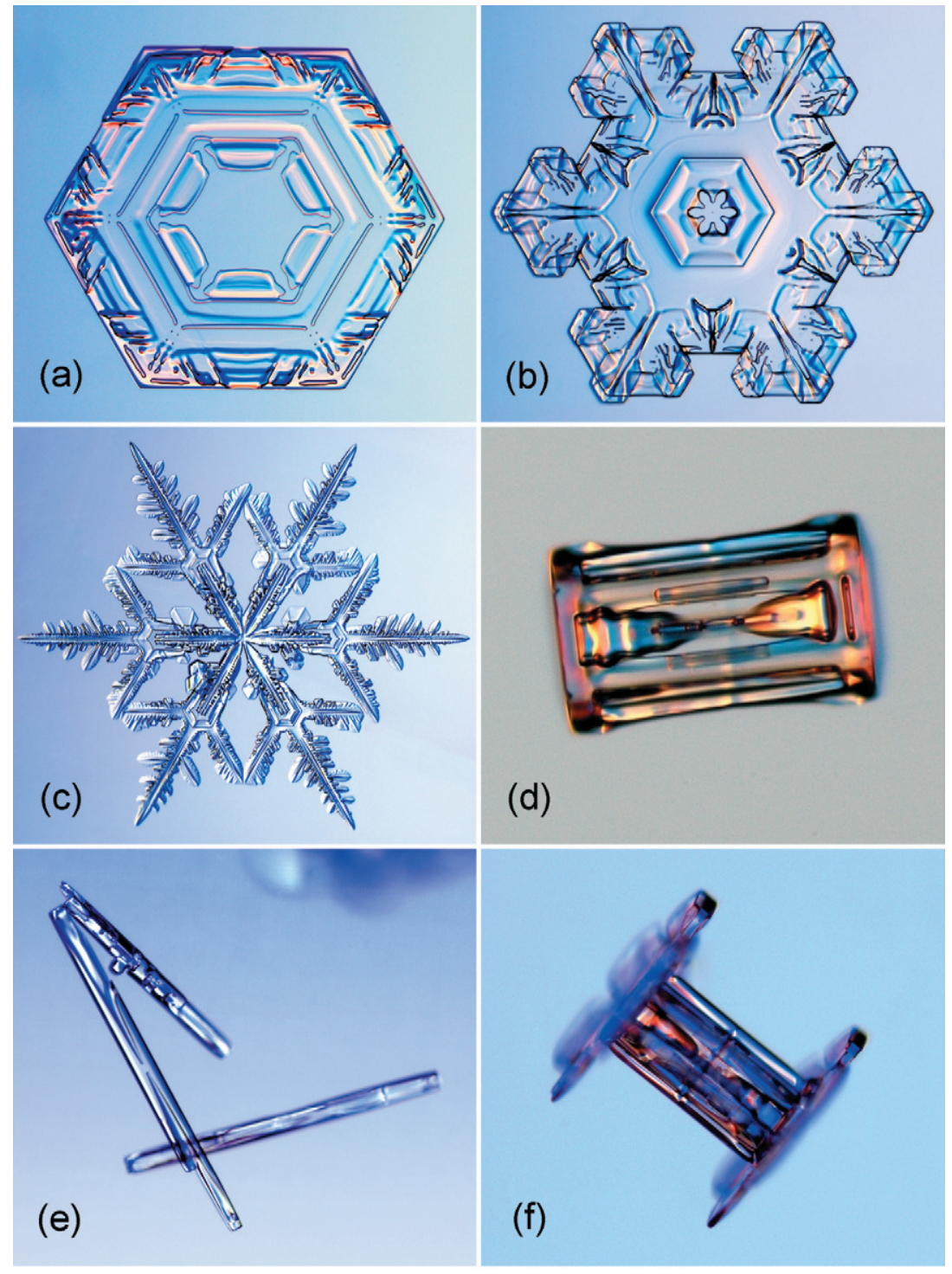

Figure 1. Examples of several different morphological types of snow crystals found in natural snowfalls in temperate climates. (a) A relatively simple plate-like crystal, $1.4 \mathrm{~mm}$ from tip to tip, showing fairly complex markings. Plates with smooth, featureless facets are less common in nature, except at low temperatures $\left(T \lesssim-20^{\circ} \mathrm{C}\right)$ and low humidities. (b) A more elaborate plate-like crystal, $2.1 \mathrm{~mm}$ from tip to tip, with an unusually high degree of complex symmetry. (c) A multi-branched stellar dendrite crystal, $3.0 \mathrm{~mm}$ from tip to tip. These typically form at higher humidities and only when the temperature is near $T=-15^{\circ} \mathrm{C}$. $(d)$ A simple hexagonal columnar crystal, $0.45 \mathrm{~mm}$ in length. Some internal hollowing resulted when the initially hollow ends of the column grew over. $(e)$ Needle-like crystals, the largest being $1.1 \mathrm{~mm}$ in length. Note one needle grew as a slender hollow column before the ends branched. These crystals only grow near $T=-5^{\circ} \mathrm{C}$. $(f)$ A capped column crystal, $0.6 \mathrm{~mm}$ in length. This crystal began growing as a stout hollow column, and then plate-like stellar crystals grew on the two ends of the column. Photos by the author [16]. 


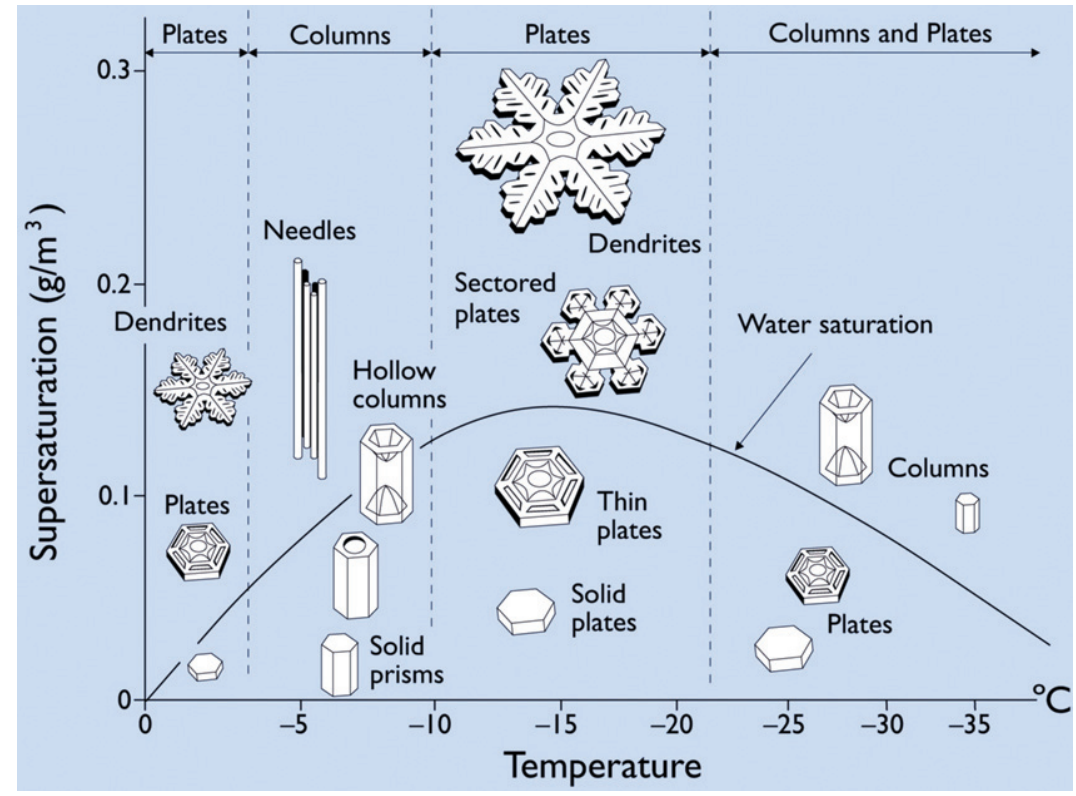

Figure 2. The snow crystal morphology diagram, showing different types of snow crystals that grow in air at atmospheric pressure, as a function of temperature and water vapour supersaturation relative to ice. The water saturation line gives the supersaturation of supercooled water, as might be found within a dense cloud. Note the morphology switches from plates $\left(T \approx-2^{\circ} \mathrm{C}\right)$ to columns $\left(T \approx-5^{\circ} \mathrm{C}\right)$ to plates $\left(T \approx-15^{\circ} \mathrm{C}\right)$ to predominantly columns $\left(T<-30^{\circ} \mathrm{C}\right)$ as temperature is decreased. Temperature mainly determines whether snow crystals will grow into plates or columns, while higher supersaturations produce more complex structures. This figure was adapted from a diagram by Furakawa.

and columns at higher supersaturations. Growth of heavy water $\left(\mathrm{D}_{2} \mathrm{O}\right)$ crystals from the vapour phase produces similar morphologies as a function of temperature, except shifted by approximately four degrees, in keeping with the isotopic shift in the freezing point between $\mathrm{D}_{2} \mathrm{O}$ and $\mathrm{H}_{2} \mathrm{O}[22]$.

Note that the morphology diagram in figure 2 is not necessarily indicative of all natural snow crystals, which are often dominated by polycrystalline forms, but rather it reflects the growth of ice single-crystals [21]. For natural crystals it is also important to note that ice growth is enhanced when air flows over a growing surface, a phenomenon called the ventilation effect [23, 24].

The variation in snow crystal morphology as a function of temperature is both dramatic and easily demonstrated. Figure 3 shows some representative laboratory-produced snow crystals, grown in free-fall in an air-filled cold chamber at temperatures of $T=-2^{\circ} \mathrm{C},-5^{\circ} \mathrm{C}$ and $-15^{\circ} \mathrm{C}$ and at intermediate supersaturations. Quantitative data of a similar nature, giving average crystal dimensions as a function of temperature and growth time, have been presented by several authors [10,25-27]. The crystals in figure 3 clearly follow the morphology diagram, growing predominantly as plates at $T=-2^{\circ} \mathrm{C}$, columns at $T=-5^{\circ} \mathrm{C}$ and plates again at $-15^{\circ} \mathrm{C}$.

One can also see a substantial variability in crystal size and shape for the crystals in figure 3, even though they were grown under fairly similar conditions. This reflects the fact that snow crystal growth is quite sensitive to small changes in temperature, supersaturation and other factors. This sensitivity is one of the hallmarks of snow crystal growth, and, as we will see later, this has made it difficult to make crystal growth measurements that are sufficiently accurate and reproducible that one can definitely discern the different growth mechanisms under 




Figure 3. Examples of laboratory-made snow crystals grown at temperatures $T=-2^{\circ} \mathrm{C}$ (left), $T=-5^{\circ} \mathrm{C}$ (middle), and $T=-15^{\circ} \mathrm{C}$ (right) and supersaturations near water saturation [19]. Vertical scale bars are $100 \mu \mathrm{m}$ long. These crystals were produced in a cold chamber filled with supersaturated air and photographed after they had fallen onto an observation window at the bottom of the chamber. Note the dramatic morphological changes from plates at $-2^{\circ} \mathrm{C}$ to columns at $-5^{\circ} \mathrm{C}$ to plates again at $-15^{\circ} \mathrm{C}$. Photos by the author [19].

different conditions. As we will see below, explaining the physics behind the snow crystal morphology diagram, even at a qualitative level, continues to be a surprisingly elusive goal.

\subsection{Complexity and symmetry}

The sensitivity of snow crystal growth to temperature and humidity allows a straightforward explanation for the combination of complexity and symmetry seen in many specimens like those shown in figure 1. Consider the formation of a single snow crystal in the atmosphere. The story begins inside a cloud, which typically consists of a large number of liquid water droplets nucleated on minute dust particles. Nucleation into liquid droplets, even at temperatures below $0^{\circ} \mathrm{C}$, is an example of Ostwald's step rule [28]. Pure water droplets of microscopic dimensions can be supercooled to temperatures as low as $T \approx-40^{\circ} \mathrm{C}$ before freezing via homogeneous nucleation. Assuming a relatively static cloud, the water droplets in the cloud will supersaturate the enclosed air to values (relative to ice) as high as the water saturation level shown in figure 1.

If the cloud temperature drops, at around $T=-10^{\circ} \mathrm{C}$ the droplets will begin to freeze, nucleated by their contained dust particles. Since some ice nucleators are better than others, the droplets will not all freeze simultaneously. A frozen droplet will quickly accumulate water vapour from the air, which stays supersaturated by virtue of the remaining unfrozen water droplets. As the nascent snow crystal grows, faceting will often create a simple hexagonal prism morphology. Diffusion limits the growth as the crystal becomes larger, and eventually this causes branches to form. Because ice growth is so sensitive to the local environment, it frequently happens that an abrupt motion of some kind will cause all six corners of a simple plate-like crystal to sprout arms at the same time. 
As the crystal travels through the cloud, it experiences different temperatures and humidities along the way, and thus the growth behaviour changes as a function of time. But of course all six arms experience the same changing conditions as they grow. The result is a rather complex growth pattern for each arm of the crystal, with all six arms developing roughly the same pattern. Under ideal conditions - for which the growth must be unperturbed by collisions with other ice or water particles - a snow crystal can grow into a rather elaborate, six-fold symmetric shape, like those shown in figure 1. As this scenario is replayed countless times, the bulk of the liquid water in a cloud is transformed into the solid state.

\subsection{The physics of snow crystals}

Many aspects of snow crystal growth are well understood at a quantitative level. For example, we know the crystal structure of ice, the interactions between water molecules, the ice phase diagram, and much of phase transitions in general. Other pieces of the snow crystal puzzle, like diffusion-limited growth and the equilibrium structure of the ice surface, are fairly well understood, at least in a qualitative sense. And then there are some rather basic aspects of this phenomenon, like the snow crystal morphology diagram, that are not yet understood even at a qualitative level.

This review will focus on two features of snow crystal growth-(1) the formation of simple, faceted prism crystals as a function of temperature and supersaturation, which we will use to examine the physics underlying the morphology diagram, and (2) the transition to, and growth of, dendritic structures. In particular, we will examine the underlying physical processes behind these phenomena, in quantitative terms as much as possible. We will call upon what is known about the detailed physics of kinetics-limited crystal growth, pattern formation in diffusion-limited solidification, and other areas, but only inasmuch as they apply to ice crystal growth.

We will not concern ourselves with the meteorological side of snow crystals to any great degree, nor will we cover the problem of ice nucleation in detail. The solidification of ice from liquid water is a related problem, with its own literature (e.g. see [29] and references therein). Here again we will only call upon this literature inasmuch as it is directly relevant to growth from the vapour phase.

A number of previous authors have reviewed the subject of snow crystal growth from a variety of perspectives [7-10,30,31]. Nakaya's classic text [9] is still a fascinating introduction to the phenomenology of snow crystals, which includes many details on the crystal morphologies of both natural and synthetic varieties. The review by Kobayashi and Kuroda is also an excellent introduction to the physics of snow crystals, although it has become somewhat dated. A nonmathematical version of snowflake science, complete with a large collection of snowflake photographs, is given in [19].

\section{Prism growth from vapour-theory}

Ice Ih, the normal form of ice, has a hexagonal crystal structure, so the basic ice crystal growth form is the hexagonal prism shown in figure 4. Cubic ice, ice Ic, has nearly the same binding energy as Ice Ih, and there is evidence that the cubic form plays a role in the nucleation of snow crystals, especially twinned and polycrystalline forms $[10,32]$. This role is relatively minor, however, so we will mostly ignore cubic ice in this review. The other solid phases of ice all appear at high pressures and/or low temperatures [33], so these too are beyond the scope of this review. 

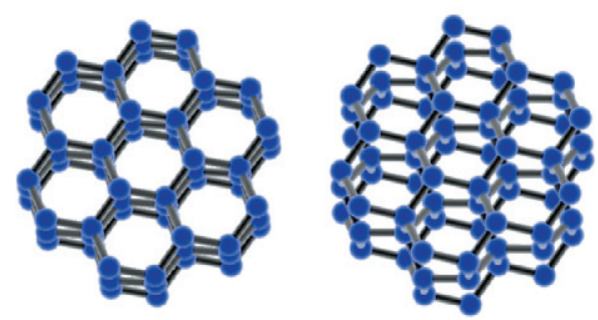
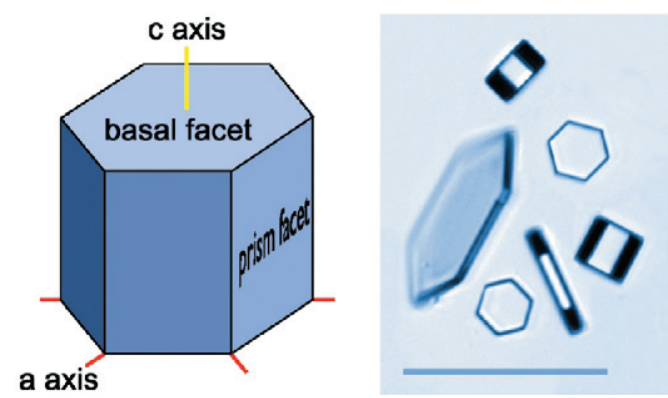

Figure 4. Left: Two views of the crystal structure of ice Ih, showing a lattice of 'puckered' hexagons. Here balls represent oxygen atoms and bars represent hydrogen atoms. Middle: A schematic picture of a simple ice prism, defining the principal crystal axes and facet planes. Right: A mosaic image of some typical small ice prisms grown in the lab, showing different aspect ratios. The scale bar is $100 \mu \mathrm{m}$ long.

Snow crystals often grow as simple hexagonal prisms, like many of those seen in figures 3 and 4, especially when the crystals are $10-100 \mu \mathrm{m}$ in size. The overall morphology can be either plate-like or columnar, depending on the relative growth rates of the basal and prism facets. Figure 3 demonstrates that even simple hexagonal prism crystals clearly exhibit the temperature dependence seen in the morphology diagram. Therefore, the first step towards understanding snow crystal formation will be to understand the growth dynamics of simple ice prisms.

\subsection{Basic terminology}

For an ice crystal growing from water vapour, we can write the growth velocity normal to the surface in terms of the Hertz-Knudsen formula [34]

$$
\begin{aligned}
v_{\mathrm{n}} & =\alpha \frac{c_{\text {sat }}}{c_{\text {solid }}} \sqrt{\frac{k T}{2 \pi m}} \sigma_{\text {surf }} \\
& =\alpha v_{\text {kin }} \sigma_{\text {surf }},
\end{aligned}
$$

where the latter defines the velocity $v_{\text {kin }}$. In this expression $k T$ is Boltzmann's constant times temperature, $m$ is the mass of a water molecule, $c_{\text {solid }}=\rho_{\text {ice }} / m$ is the number density for ice, $\sigma_{\text {surf }}=\left(c_{\text {surf }}-c_{\text {sat }}\right) / c_{\text {sat }}$ is the supersaturation just above the growing surface, $c_{\text {surf }}$ is the water vapour number density at the surface, and $c_{\text {sat }}(T)$ is the equilibrium number density above a flat ice surface. The parameter $\alpha$ is known as the condensation coefficient, and it embodies the surface physics that governs how water molecules are incorporated into the ice lattice, collectively known as the attachment kinetics. The attachment kinetics can be nontrivial, so in general $\alpha$ will depend on $T, \sigma_{\text {surf }}$, the surface structure and geometry, surface chemistry, etc. If molecules striking the surface are instantly incorporated into it, then $\alpha=1$; otherwise we must have $\alpha \leqslant 1$. The appearance of crystal facets indicates that the growth is limited by attachment kinetics, so we must have $\alpha<1$ on faceted surfaces. For a molecularly rough surface, or for a liquid surface, we expect $\alpha \approx 1$ [35].

One should note that the parameterization in terms of $\alpha$ assumes that the attachment kinetics can be described as an intrinsically local process, which may not always be a valid assumption. For the ideal case of an infinite, defect-free surface this parameterization must be valid, since in this limit it is little more than a mathematical definition. Nonlocal effects, however, such as transport between facets, could make the parameterization invalid in some circumstances. For example, surface diffusion is lumped into $\alpha$, which effectively implies that 
the diffusion distance of adsorbed molecules on the ice surface (before they are incorporated into the lattice) is much smaller than a characteristic crystal size. On a thin, plate-like snow crystal, however, it is not impossible that surface diffusion carries adsorbed water molecules from the basal facets to the prism facets, thereby enhancing growth of the prism facets, to the point that equation (1) would yield $\alpha_{\text {prism }}>1$. If this happens, then the parameterization in terms of $\alpha$ is invalid and surface diffusion must be specifically included in the overall problem if one is to understand the growth dynamics. The Schwoebel-Ehrlich effect provides a potential barrier that inhibits surface diffusion around corners [34,36], and there is experimental evidence that supports $\alpha<1$ for snow crystal growth (see below). Therefore the assumption of purely local attachment kinetics, parameterized by the condensation coefficient, will pervade our subsequent discussion.

We note at this point that the equilibrium crystal shape-that which minimizes the total surface energy - plays only a minor role when considering the growth of snow crystals. To see why this is, consider the evolution of an isolated crystal spheroid and assume $\alpha \approx 1$. The equilibrium vapour pressure at each point on the surface will depend on surface curvature owing to the Gibbs-Thomson effect [34], so $c(R)=c_{\text {sat }}(1+2 \delta / R)$, where $\delta=\gamma / c_{\text {solid }} k T \approx 1 \mathrm{~nm}$, $\gamma$ is the ice surface energy, and $R$ is the local radius of curvature of the surface. The timescale for a spheroid to revert to a sphere is then roughly $\tau_{\text {equil }} \approx R^{2} / v_{\text {kin }} \delta \approx 200 \cdot[R /(10 \mu \mathrm{m})]^{2}$ seconds near the melting point. We see that this time is quite long for all but the smallest crystals. Under most snow crystal growth conditions the timescale for reaching the equilibrium shape is much longer than the timescale for growth. Thus the surface energy term usually plays a negligible role in determining the aspect ratios of simple prisms.

To my knowledge the equilibrium shapes of isolated ice crystals have never been definitively observed. As a result, $\Delta \gamma=\gamma_{\text {basal }}-\gamma_{\text {prism }}$ has not been measured, although one expects on theoretical grounds that $\Delta \gamma / \gamma \lesssim 0.01$ [10]. Thus the equilibrium shape would be nearly spherical with only slight faceting [37]. Faceted areas generally become larger during growth and smaller during sublimation. Thus even small crystals growing sufficiently fast become essentially completely faceted, like those shown in figure 4 [37, 38].

Note that ice crystal growth from the vapour is almost always in a regime where $\sigma_{\text {surf }} \ll c_{\text {sat }}$. This is in contrast to thin-film deposition, where often $c_{\text {sat }}$ is so small as to be negligible. Thus $v_{\text {kin }}$ can be thought of as a 'refresh rate' for the surface-the rate at which the surface is simultaneously evaporating and regrowing. The velocity $v_{\mathrm{n}}$ represents the net growth that occurs. Since $v_{\mathrm{n}} \ll v_{\text {kin }}$, an ice facet cannot be thought of as a static crystal plane, like something that might be slowly imaged by a scanning probe microscope. Rather the surface is a very dynamical place, where molecules in the solid phase are being very rapidly exchanged with those from the surrounding vapour.

\subsection{Diffusion-limited growth}

Snow crystal growth dynamics is typically dominated by attachment kinetics in combination with two transport effects: particle diffusion, which carries water molecules to the growing crystal, and heat diffusion, which removes latent heat generated by solidification. The interplay of these three processes is ultimately responsible for the vast diversity of snow crystal morphologies, so the task at hand is to understand how they each affect crystal growth. The transport effects - particle and heat diffusion - are well understood at a fundamental level, so in principle we can compute how they limit crystal growth. In practice, of course, this may be difficult owing to the complex geometry of the solidification front.

Attachment kinetics, by contrast, is very much not yet understood in detail, and numerous experiments on ice crystal growth have been aimed at exploring the molecular dynamics that 
occurs at a growing ice surface. For these experiments it is necessary to disentangle the different mechanisms limiting crystal growth, to model particle and heat diffusion at a sufficient level that the attachment kinetics can be extracted with confidence. Thus before going further we must understand particle and heat diffusion as they apply to ice crystal growth.

Particle transport is described by the diffusion equation

$$
\frac{\partial c}{\partial t}=D \nabla^{2} c
$$

where $c(x)$ is the water molecule number density surrounding the crystal and $D$ is the diffusion constant. The timescale for diffusion to adjust the vapour concentration in the vicinity of a crystal is $\tau_{\text {diffusion }} \approx R^{2} / D$, where $R$ is a characteristic crystal size. This is to be compared with the growth time, $\tau_{\text {growth }} \approx 2 R / v_{\mathrm{n}}$, where $v_{\mathrm{n}}$ is the growth velocity of the solidification front normal to the surface. The ratio of these two timescales is the Peclet number, $p=R v_{\mathrm{n}} / 2 D$. For typical growth rates of snow crystals we find $p \lesssim 10^{-5}$, which means that diffusion adjusts the particle density around the crystal much faster than the crystal shape changes. In this case the diffusion equation reduces to Laplace's equation, $\nabla^{2} c=0$, which must be solved with the appropriate boundary conditions. Using this slow-growth limit simplifies the problem considerably in comparison to much of the literature on diffusion-limited solidification.

The continuity equation at the interface gives

$$
v_{\mathrm{n}}=\frac{D}{c_{\text {solid }}}(\hat{n} \cdot \vec{\nabla} c)_{\text {surf }}=\frac{c_{\text {sat }} D}{c_{\text {solid }}}(\hat{n} \cdot \vec{\nabla} \sigma)_{\text {surf }},
$$

where $\sigma(x)=\left[c(x)-c_{\text {sat }}\right] / c_{\text {sat }}$ and we are assuming the isothermal case to begin, so $c_{\text {sat }}$ is independent of spatial position.

Before attempting to solve the diffusion equation for complex crystal geometries, it is instructive to first consider the growth of a fictitious 'faceted' spherical crystal, where the equations reduce to one dimension and can be solved analytically. In this approximation we treat the growing spherical surface as if it had the same attachment kinetics as a flat faceted surface, with some arbitrary $\alpha$ that is constant around the sphere. This one-dimensional treatment contains much of the physics found with simple prism geometries, but of course it is much easier to solve and to visualize. We consider this problem in three limiting cases of increasing difficulty.

2.2.1. Case I-finite kinetics $(\alpha \leqslant 1)$ without heating. In the absence of heating we can assume a constant temperature, independent of position, and a straightforward solution of the diffusion equation yields the growth velocity [39]

$$
\begin{aligned}
v_{\mathrm{n}} & =\frac{\alpha \alpha_{\mathrm{diff}}}{\alpha+\alpha_{\mathrm{diff}}} v_{\mathrm{kin}} \sigma_{\infty} \\
& =\frac{\alpha}{\alpha+\alpha_{\mathrm{diff}}} \frac{c_{\mathrm{sat}} D \sigma_{\infty}}{c_{\mathrm{solid}} R},
\end{aligned}
$$

where

$$
\alpha_{\text {diff }}=\frac{c_{\text {sat }} D}{c_{\text {solid }} v_{\text {kin }} R}=\frac{D}{R} \sqrt{\frac{2 \pi m}{k T}},
$$

$v_{\text {kin }}$ was defined in equation (1), $\sigma_{\infty}$ is the supersaturation far from the growing crystal, and $R$ is the sphere radius.

In the limit $\alpha_{\text {diff }} \ll \alpha$ the growth velocity becomes $v_{\mathrm{n}}=c_{\text {sat }} D \sigma_{\infty} / c_{\text {solid }} R$, which describes purely diffusion-limited growth in which attachment kinetics can be neglected. In the opposite 
limit we have $v_{\mathrm{n}}=\alpha v_{\text {kin }} \sigma_{\infty}$, which is valid for purely kinetics-limited growth unhindered by particle transport. For the specific case of ice growing at $T=-15^{\circ} \mathrm{C}$ in air we have

$$
\alpha_{\text {diff }}\left(-15^{\circ} \mathrm{C}\right) \approx 0.15\left(\frac{1 \mu \mathrm{m}}{R}\right)\left(\frac{D}{D_{\text {air }}}\right),
$$

where $D_{\text {air }} \approx 2 \times 10^{-5} \mathrm{~m}^{2} \mathrm{~s}^{-1}$ is the diffusion constant for water vapour in air at a pressure of 1 atm.

By writing the growth velocity in this form it becomes apparent that one cannot easily use measurements of the growth velocity $v_{\mathrm{n}}$ to determine $\alpha$ if $\alpha_{\text {diff }} \lesssim \alpha$. In this case the growth is mostly diffusion limited, and an examination of equation (4) reveals that small errors in the determination of $v_{\mathrm{n}}$ can result in large errors in the derived $\alpha$. To measure $\alpha$ using crystal growth measurements we must either use very small crystals or reduce the background gas pressure (since typically $D \sim P^{-1}$, where $P$ is the background pressure).

2.2.2. Case II-diffusion-limited growth $\left(\alpha_{\text {diff }} \ll \alpha\right)$ with heating. In this case we must solve the double diffusion problem, considering both particle diffusion to the growing crystal and thermal diffusion to remove latent heat generated at the interface. The spherical case can again be solved exactly in the slow-growth limit [39], yielding

$$
v_{\mathrm{n}}=\frac{D}{R} \frac{c_{\mathrm{sat}}}{c_{\text {solid }}} \frac{\sigma_{\infty}}{1+\chi_{0}}
$$

where we use the convention that $c_{\text {sat }}=c_{\text {sat }}\left(T_{\infty}\right)$. In this expression we have defined

$$
\chi_{0}=\frac{\eta D \lambda \rho_{\text {ice }}}{\kappa} \frac{c_{\text {sat }}}{c_{\text {solid }}},
$$

where $\lambda=2.8 \times 10^{6} \mathrm{~J} \mathrm{~kg}^{-1}$ is the latent heat for the vapour/solid transition, $\rho_{\text {ice }}=917 \mathrm{~kg} \mathrm{~m}^{-3}$ is the solid density, $\kappa$ is the thermal conductivity of the solvent gas $\left(\kappa \approx 0.025 \mathrm{~W} \mathrm{~m}^{-1} \mathrm{~K}^{-1}\right.$ for air), and $\eta=\mathrm{d} \log \left(c_{\mathrm{sat}}\right) / \mathrm{d} T$. Typical values for these parameters are

\begin{tabular}{lllll}
\hline$T\left({ }^{\circ} \mathrm{C}\right)$ & $c_{\text {sat }} / c_{\text {solid }}$ & $v_{\text {kin }}\left(\mu \mathrm{m} \mathrm{s}^{-1}\right)$ & $\eta$ & $\chi_{0}$ \\
\hline-40 & $0.13 \times 10^{-6}$ & 17 & 0.11 & 0.03 \\
-30 & $0.37 \times 10^{-6}$ & 49 & 0.10 & 0.08 \\
-20 & $0.96 \times 10^{-6}$ & 131 & 0.092 & 0.18 \\
-15 & $1.51 \times 10^{-6}$ & 208 & 0.088 & 0.27 \\
-10 & $2.33 \times 10^{-6}$ & 324 & 0.085 & 0.41 \\
-5 & $3.54 \times 10^{-6}$ & 496 & 0.082 & 0.59 \\
-2 & $4.51 \times 10^{-6}$ & 635 & 0.080 & 0.74 \\
-1 & $4.88 \times 10^{-6}$ & 689 & 0.079 & 0.79 \\
\hline
\end{tabular}

where $\chi_{0}$ was evaluated in air at a pressure of $1 \mathrm{~atm}$. We see that the main effect of heating on diffusion-limited growth is to scale the growth by a factor of $\left(1+\chi_{0}\right)^{-1}$. In near-vacuum conditions the diffusion constant is large, so that $\chi_{0} \gg 1$ and the growth velocity becomes limited mainly by heating. In this limit we have

$$
\begin{aligned}
v_{\mathrm{n}} & \approx \frac{\kappa}{\lambda \rho \eta} \frac{\sigma_{\infty}}{R} \\
& \approx\left(110 \mu \mathrm{m} \mathrm{s}^{-1}\right)\left(\frac{1 \mu \mathrm{m}}{R}\right) \sigma_{\infty},
\end{aligned}
$$

where the latter is evaluated at $T=-15^{\circ} \mathrm{C}$. 
2.2.3. Case III-finite kinetics, with heating. For the most general case, with finite kinetics in addition to heating, the analysis is similar to the above and the final result is [39]

$$
v_{\mathrm{n}} \approx \frac{\alpha}{\alpha\left(1+\chi_{0}\right)+\alpha_{\text {diff }}} \frac{c_{\text {sat }} D \sigma_{\infty}}{c_{\text {solid }} R} .
$$

This can be rewritten as

$$
v_{\mathrm{n}} \approx \frac{\alpha^{*}}{\alpha^{*}+\alpha_{\mathrm{diff}}} \frac{c_{\mathrm{sat}} D \sigma_{\infty}^{*}}{c_{\mathrm{solid}} R}
$$

where $\alpha^{*}=\alpha\left(1+\chi_{0}\right)$ and $\sigma_{\infty}^{*}=\sigma_{\infty} /\left(1+\chi_{0}\right)$, and we note the similarity to the solution in Case I. Again the main effect of heating is to scale the growth by the factor $\left(1+\chi_{0}\right)^{-1}$.

If the diffusion constant is large, this becomes

$$
v_{\mathrm{n}} \approx \frac{\alpha \alpha_{\text {cond }}}{\alpha+\alpha_{\text {cond }}} v_{\text {kin }} \sigma_{\infty}
$$

where

$$
\begin{aligned}
\alpha_{\text {cond }} & =\frac{\kappa}{\eta R \lambda \rho v_{\text {kin }}} \\
& \approx 0.5\left(\frac{1 \mu \mathrm{m}}{R}\right),
\end{aligned}
$$

where the latter expression is again evaluated at $T=-15^{\circ} \mathrm{C}$. We note here that $\kappa$ is roughly independent of background pressure down to fairly low pressures.

2.2.4. Substrate simulation. We can also use the spherical solution to examine growth on a substrate if we assume a hemispherical crystal with the flat surface held at the substrate temperature $T_{\text {substrate }}$ [39]. This gives

$$
v \approx \frac{\alpha}{\alpha\left(1+\chi_{0}^{\prime}\right)+\alpha_{\text {diff }}} \frac{c_{\mathrm{sat}} D \sigma_{0}}{c_{\mathrm{solid}} R},
$$

where

$$
\chi_{0}^{\prime}=\frac{2 \eta D \lambda \rho}{G \kappa_{\text {ice }}} \frac{c_{\text {sat }}}{c_{\text {solid }}}
$$

and $G \approx 1$ is a geometrical factor. In the case of fast diffusion this becomes

$$
v=\frac{\alpha \alpha_{\text {cond }}^{\prime}}{\alpha+\alpha_{\text {cond }}^{\prime}} v_{\text {kin }} \sigma_{0}
$$

with

$$
\alpha_{\text {cond }}^{\prime}=\frac{G \kappa_{\text {ice }}}{2 \eta R \lambda \rho v_{\text {kin }}} .
$$

At $T=-15^{\circ} \mathrm{C}$ this becomes

$$
\alpha_{\text {cond }}^{\prime} \approx 25 G\left(\frac{1 \mu \mathrm{m}}{R}\right) .
$$

Since $\kappa_{\text {ice }} / \kappa_{\text {air }} \approx 100$, the presence of a substrate reduces the temperature increase of the growing crystal by a factor of $\sim 100$ when compared to free growth in air. 
2.2.5. Relation to experiments. The analytic results obtained above for a growing sphere are useful for gauging the relative importance of diffusion and attachment kinetics, and are especially useful when designing or interpreting experiments aimed at measuring $\alpha$ from the growth of simple prisms. For example, if one wants to measure $\alpha$ for crystals growing in a background gas, then it is necessary to achieve a condition where $\alpha<\alpha_{\text {diff }}$. Diffusion modelling will not help much if this inequality is not met, because small systematic errors in the measured $v_{\mathrm{n}}$ will invariably amplify into large errors in the inferred $\alpha$. Similarly, crystal growth on a substrate must be done under conditions where $\alpha<\alpha_{\text {cond }}^{\prime}$, since otherwise thermal diffusion will distort the results. As we will see below, these are not trivial considerations and many ice growth experiments have produced unreliable data because of systematic effects of this nature. Modern ice growth experiments are just beginning to address and eliminate these systematic errors, and are thus just beginning to produce data of sufficient accuracy to shed light on the microphysics that underlies the snow crystal morphology diagram.

\subsection{Diffusion modelling of prism growth}

For real ice crystals, which are almost certainly nonspherical, one must employ more sophisticated techniques to model diffusion-limited growth. For the case of simple hexagonal prisms, for which $v_{\mathrm{n}}$ is constant across a facet, a numerical solution of the diffusion equation is quite tractable using commercial codes. While it is possible to solve the three-dimensional Laplace equation around a hexagonal prism [40], there is much to be gained by looking first at a two-dimensional cylindrical approximation, which is much simpler to solve and easier to visualize. In this approximation the hexagonal prism is replaced by a solid cylinder, where the six prism facets are approximated as one fictitious curved 'facet' that is capped by two circular basal facets. This approximation is more than adequate for comparing with experiments measuring the growth of snow crystal prisms, in part because the growth data to date have not been very precise.

For many cases of simple prism growth, the double problem of heat and particle diffusion reduces to primarily a particle diffusion problem only, as seen in the discussion of equation (11). The thermal conductivity of ice is high enough, and the heating rate from solidification is low enough, that heating tends to raise the crystal temperature uniformly. This affects the growth by effectively changing $c_{\text {sat }}$, which can be modelled as simply a change in $\sigma_{\infty}$, even when the crystal geometry is nontrivial. A reasonable estimate of the crystal temperature rise can often be obtained from a spherical analysis, with the total rate of mass increase as input. This rough estimation of heating may not be sufficient for all circumstances, especially crystal growth at low background pressures [41]. But in many practical circumstances the double diffusion problem reduces in essence to particle diffusion only.

For the particle diffusion problem one must solve Laplace's equation in the space surrounding the crystal, and there are several ways to specify the boundary conditions at the crystal surface. The first choice is to specify the surface supersaturation $\sigma_{\text {surf }}$ over the entire crystal. If $\alpha \approx 1$ and $v_{\mathrm{n}} \ll v_{\text {kin }}$, then equation (1) suggests that $\sigma_{\text {surf }} \approx 0$ is a reasonable approximation for the surface condition. For most ice prisms, however, $\alpha \ll 1$ on the facets and $\sigma_{\text {surf }} \approx 0$ is not a good approximation. In such cases one does not know $\sigma_{\text {surf }}$ on the surface, so a different choice of boundary conditions is necessary.

The second possibility is to specify $\alpha \sim \sigma^{-1} \nabla_{\mathrm{n}} \sigma$ over the entire crystal surface. This is often impractical for crystal prisms because $\alpha$ varies considerably over a facet surface, as we shall see. The third choice for boundary conditions is to specify the growth velocity $v_{\mathrm{n}} \sim \nabla_{\mathrm{n}} \sigma$ at the surface. This choice is especially useful for comparing with experiments on prism growth, since $v_{\mathrm{n}}$ is a measured quantity. Furthermore, as long as the crystal growth morphology remains 
that of a simple hexagonal prism, then we must have that $v_{\mathrm{n}}=$ constant over an entire facet. Thus specifying $v_{\mathrm{n}}$ is often the most practical choice of boundary conditions at the ice surface. The boundary condition far from the crystal is nearly always to specify $\sigma_{\infty}$.

Commercial diffusion codes are typically adapted for heat diffusion, but they can be used for solving the particle diffusion problem as well. In this application one uses the temperature field $T(x)$ as a surrogate for the supersaturation $\sigma(x)$, and one can easily work out the proper conversion factors for the specific code. In a heat diffusion code the above choices for boundary conditions are called normal boundary conditions, convective boundary conditions and heat flux boundary conditions, respectively, and again working out the proper conversion factors is a straightforward exercise.

We examine the full problem of modelling diffusion-limited growth in more detail below, including pattern formation, but make a few passing comments here. First, Green's function methods can be used to convert a two-dimensional cylindrical problem to one-dimensional, which then allows simpler numerical solution [42,43]. These techniques can be useful for generating code tailored to a specific modelling problem, for which commercial codes may not be practical. Second, it is possible in principle to model the growth of an ice crystal as a function of time by front-tracking methods: computing $v_{\mathrm{n}}$ around the crystal, advancing the interface for a small time-step, recalculating $v_{\mathrm{n}}$, etc [44]. This turns out to be an exceedingly tricky task in practice, even for simple faceted crystals, because the attachment kinetics are such a strong function of surface orientation relative to the crystal axes. The movement of small steps on the surface is especially problematic in this regard, and to date the results have not been very satisfying. Third, these simple diffusion calculations do not include ventilation effects, which describe the enhanced growth of snow crystals in the presence of a moving airstream [23]. Such effects are typically negligible for small prisms with sizes less than $100 \mu \mathrm{m}$, but may be important when the structures are larger.

\subsection{Facet growth with diffusion}

Having examined the basic tools for two-dimensional diffusion modelling, it is instructive to consider some simple cases of prism growth, especially with different aspect ratios. Figure 5 shows some characteristic calculations obtained using a commercial diffusion code [45] for growing ice prisms. Here we used velocity boundary conditions because the prism morphology does not change appreciably during growth and $v_{\mathrm{n}}$ is constant over a facet, as mentioned above. One immediately sees in figure 5 that $\sigma_{\text {surf }}$ varies considerably over the ice surface, and that there are three distinct cases of prism growth.

The first case is for roughly isometric crystals, where the growth rates of the basal and prism facets are comparable. The solution of the diffusion equation immediately implies that $\sigma_{\text {surf }}$ is a maximum at the edges of the facets, as seen in figure 5. Since $v_{\mathrm{n}}$ is constant over a facet, equation (1) requires that $\alpha \sigma_{\text {surf }}$ must be constant as well, meaning $\alpha$ is largest at the centres of the facets. This in turn implies the facets cannot be completely flat at the molecular level, and are instead concave to some degree. The concave structure of each facet is such that the step density, and thus $\alpha$, is highest at the facet centre. Steps are then likely generated from two-dimensional nucleation at the facet edges, and the steps propagate inward as the crystal grows. For larger crystals, diffusion brings about larger variations in $\sigma_{\text {surf }}$ across a facet. Eventually this leads to the branching instability described below.

The second case is that of thin plate crystals, which is the second example shown in figure 5 . Here the growth is such that $\sigma_{\text {surf }}$ is largest at the centres of the basal facets, so $\alpha$ is smallest there. Thus the basal facets are convex at the molecular scale, and basal steps are generated at the facet centres and propagate outward. Note this result follows simply from a solution of 



Figure 5. Left two panels: diffusion calculations around two growing ice prisms, to demonstrate concave and convex growth. The first (leftmost) panel shows the cross-section of a growing thick plate with diameter/thickness ratio $D / T=2$. The calculation assumed axial symmetry, so the top and bottom surfaces are the basal facets, and the left edge represents the prism facet. Velocity boundary conditions were used, with $v_{\text {prism }} / v_{\text {basal }}=2$. The contours show lines of constant supersaturation in the air around the crystal. Here we see $\sigma_{\text {surf }}$ is highest at the corners of the crystal, indicating that the faces are all concave and that $\alpha$ is highest at the centres of both facets. In this case, growth steps originate at the edges of the facets and propagate inward. The second panel shows a similar calculation with $D / T=20$ and $v_{\text {prism }} / v_{\text {basal }}=40$. This time $\sigma_{\text {surf }}$ is highest at the centres of the basal facets, indicating that these facets are convex. Thus growth steps originate at the centre of a basal surface and propagate toward the edges. This behaviour must be present for the growth of thin snow crystal plates in air at $T=-15^{\circ} \mathrm{C}$ (rightmost panel above; scale bar $50 \mu \mathrm{m})$ [27].

the diffusion equation in the space surrounding the crystal, with the facet growth velocities as inputs. The dividing line between the convex and concave cases for plate-like prism crystals occurs at diameter/thickness ratios of roughly $D / T \approx 10$, depending on assumptions about the crystal geometry. Both these cases are commonly seen in the growth of natural and synthetic snow crystals.

The third case, not shown in figure 5, is that of thin columnar growth with $D / T \ll 1$. In this case the prism facets will become convex, which again follows directly from a solution of the diffusion equation. It appears this case does not readily occur in natural snow crystals because branching tends to happen before convex prism facets develop. However, the tips of $c$-axis electric needle crystals (see below) probably do exhibit convex prism surfaces.

\subsection{Attachment kinetics}

The above treatments of particle and heat diffusion are typically sufficient for analysing the growth of hexagonal ice prisms to an adequate precision, since the prism geometry is relatively simple. What remains is to examine the condensation coefficient $\alpha$ in detail. Much has been written about the molecular dynamics behind attachment kinetics, and here we only briefly mention the basics that likely apply to snow crystal growth.

The simplest case is that of a molecularly rough crystal surface, which has the highest possible density of kink sites [34]. On such a surface the admolecules are indistinguishable from surface molecules, which often means $\alpha \approx 1$. There is little hard experimental evidence to support the notion that $\alpha \approx 1$ on a rough ice surface, but much effort has gone into measuring $\alpha$ for liquid water surfaces. Many early experiments suggested $\alpha<1$ for water, but subsequent work showed that evaporative cooling was highly significant in those experiments, making the 
early results unreliable. There is a rough consensus now that $\alpha \approx 1$ for a liquid water surface, although this is not universally accepted [46]. We will usually assume $\alpha \approx 1$ for a molecularly rough ice surface.

2.5.1. Nucleation-limited growth. The next simplest case to consider is that of a molecularly flat facet surface, or terrace [34]. Steps and kinks are not present on a perfectly flat surface, and in the absence of suitable attachment sites admolecules tend to evaporate off before becoming incorporated into the surface. Growth then only occurs at an appreciable rate when the admolecule density is sufficiently high that two-dimensional islands can nucleate on the surface. Once an island forms, it grows because its edges provide steps and kinks for incorporating new admolecules. The resulting growth rate $v_{\mathrm{n}}$ depends on the nucleation rate as well as the island growth rate. If the former is very low, then each island grows out completely before the next forms, resulting in layer-by-layer growth. This regime appears to be of little relevance for snow crystal growth.

For higher nucleation rates, there may be many growing islands on a surface at once, and islands growing on top of other islands. This situation has been analyzed in numerous 'birthand-spread' models $[35,47]$, giving results that depend in detail on numerous assumptions made in the models. Birth-and-spread models typically yield growth rates that are parametrized [34]

$$
\alpha\left(T, \sigma_{\text {surf }}\right) \approx A\left(T, \sigma_{\text {surf }}\right) \exp \left(-\frac{\sigma_{\text {crit }}}{\sigma_{\text {surf }}}\right)
$$

where $\sigma_{\text {crit }}$ is called the critical supersaturation, given by

$$
\sigma_{\text {crit }}=\frac{\pi \beta^{2} \Omega_{2}}{3(k T)^{2}},
$$

where $\beta$ is the edge free energy for a growth island that is one molecular layer thick and $\Omega_{2}$ is the area taken by a molecule on the surface. $A$ is a complicated parameter that derives mainly from the mean step velocity, which in turn depends on the mean surface diffusion distance, the admolecule residence time, and other factors. In general, $A$ depends weakly on supersaturation, and for comparison with current experiments we can approximate $A\left(T, \sigma_{\text {surf }}\right) \approx A(T)$. As we will see below, experiments show that the formation of faceted ice prisms is often dominated by nucleation-limited growth.

2.5.2. Growth mediated by dislocations and other step sources. Anything that provides a source of molecular steps can influence the growth of a faceted surface. Examples include impurities that reside on the surface, the intersection of a facet surface with a foreign object, or crystal imperfections. Screw dislocations are an especially important source, since these can provide a continuous train of steps that propagate across the surface [34]. When $\sigma_{\text {surf }} \ll \sigma_{\text {crit }}$, island nucleation is rare and the resulting growth is typically dominated by the influence of dislocations and other step sources. For the case of screw dislocations, the resulting growth rates go as $v_{\mathrm{n}} \sim \sigma_{\text {surf }}^{2}$, or equivalently $\alpha \sim \sigma_{\text {surf }}$. This mechanism is often important in the growth of ice prisms at low supersaturations.

\subsection{Surface structure and crystal growth}

Attachment kinetics ultimately derives from the molecular dynamics present at a crystal surface, and the dynamics is intimately coupled to the molecular structure of the surface. In the case of ice, numerous studies have found that the surface structure is both complex and quite temperature dependent near the melting point. It is further generally accepted that 
changes in surface structure must somehow be responsible for the dramatic changes in snow crystal growth with temperature. One thing that makes snow crystal physics so intriguing is that we do not yet understand the ice surface structure very well, nor its impact on growth, and thus we still cannot explain the snow crystal morphology diagram.

Crystalline surfaces frequently do not have the same molecular structure as in the bulk crystal. Instead, various forms of surface reconstruction take place to lower the surface energy. Near the bulk melting point an ice surface is a rather dynamic place, as evidenced by a high vapour pressure (and large $v_{\text {kin }}$ ). Thus scanning probe microscopy cannot image the surface structure directly and we must rely on more indirect surface probes to gather information. In many ways, measurements of ice crystal growth rates are an excellent surface probe, because $\alpha$ is so sensitive to surface structure. To use this probe, however, we must understand the complete problem - how the ice surface changes with temperature and other parameters, and how these changes are reflected in the attachment kinetics.

2.6.1. Surface roughening. For a crystal at finite temperature, there are two contributions to the surface free energy, $F=E-T S$. The first is the binding energy, which favours a smooth surface with the least number of dangling bonds, and the second is the surface entropy, which favours a rougher surface with the greatest number of possible configurations. At low temperatures the binding energy wins and the equilibrium structure is close to a flat, faceted surface. Near the melting point, however, it becomes favourable for the surface to contain a greater number of molecular edges and kinks [34]. Depending on the details of the molecular structure of the crystal, there may exist a roughening temperature $T_{\mathrm{R}}$, above which the surface becomes completely rough.

Much has been written about the roughening transition, in part because of its mathematical simplicity [34, 48,49], and also because surface roughening is observed in many real crystals. Without measuring the surface structure directly (which is difficult for a high vapour pressure material like ice), there are two indirect observations that are likely indicators of surface roughening: (1) above $T_{\mathrm{R}}$ the attachment kinetics are described by $\alpha \approx 1$, and (2) the growth form is nonfaceted even at low supersaturations. The evidence for surface roughening in ice will be discussed in the experimental section below.

Sometimes a faceted surface will exhibit a relatively sharp transition from $\alpha<1$ to $\alpha \approx 1$ as $\sigma$ is increased, even if it is not above its equilibrium roughening temperature. This may be an indication of kinetic roughening, in which surface roughening is induced by rapid growth [50]. Kinetic roughening is not a well-defined thermodynamic phase transition, and it occurs when island formation on the surface is relatively easy. As with static surface roughening, kinetic roughening precludes the formation of crystal facets. This phenomenon has not been studied much in ice crystal growth, but it may be important for the formation of ice dendrites, which occurs at high supersaturations.

2.6.2. Surface melting. In a sense, surface melting is a more developed form of surface roughening. The molecules near the free surface of a solid are not as tightly bound as molecules in the bulk, and for many materials a thin, quasi-liquid layer forms on the solid surface at temperatures slightly below the bulk melting point $T_{\mathrm{m}}$. The width of the melted layer diverges as the temperature approaches $T_{\mathrm{m}}$, thus making it impossible to superheat the solid phase above the bulk melting point. This phenomenon is called surface melting, and there is ample evidence that ice exhibits surface melting at temperature above roughly $-15^{\circ} \mathrm{C}$ (for a summary of data pertaining to ice, see $[33,51]$; for a general review of surface melting, see [52]). 
One can estimate the temperature-dependent thickness of the quasi-liquid layer in a physically intuitive way using the Lindemann criterion, which states that a solid will melt if the thermal fluctuations of the intermolecular distance become larger than approximately $10-15 \%$ of the average distance [53]. This century-old empirical melting criterion works reasonably well for a wide range of bulk materials [54], and it has been directly verified both experimentally [55] and in molecular dynamics simulations [56]. If we assume a simple model in which a molecule is held in a harmonic potential, then intermolecular fluctuations have an amplitude of approximately

$$
x=\left(\frac{k T}{\kappa}\right)^{1 / 2},
$$

where $\kappa$ is an effective spring constant. Near the solid surface the molecular binding is weaker than in the bulk, so we write

$$
\kappa(d)=\kappa_{0}-f(d) \Delta \kappa,
$$

where $d$ is the distance from the surface, $\Delta \kappa$ is a constant, and $f(d)$ is a function with $f(0)=1$ and $f(\infty)=0$. The potential is weaker at the surface, so $\Delta \kappa>0$. However, the potential comes mainly from nearest-neighbour interactions, so we expect $\Delta \kappa \ll \kappa_{0}$.

The Lindemann criterion states that the bulk will melt when $x$ reaches some value $x_{0}=\left(k T_{\mathrm{m}} / \kappa_{0}\right)^{1 / 2}$. Extending this, we expect the onset of surface melting will occur down to a depth $d$ at which

$$
x(d)=\left(\frac{k T}{\kappa_{0}-f(d) \Delta \kappa}\right)^{1 / 2}=x_{0} .
$$

Expanding for small $\Delta \kappa / \kappa_{0}$ and for small $t=\left(T_{\mathrm{m}}-T\right) / T_{\mathrm{m}}$, where $T_{\mathrm{m}}$ is the bulk melting temperature, this reduces to simply

$$
t=\frac{f(d) \Delta \kappa}{\kappa_{0}} .
$$

It is customary in the case of ice to assume $f(d)=\exp (-d / \lambda)$, where $\lambda$ is a scaling length for the intermolecular interactions, and with this the thickness of the quasi-liquid layer becomes

$$
d(T)=\lambda \log \left(\frac{\Delta \kappa}{t \kappa_{0}}\right),
$$

which is valid for small $t$.

It is instructive to compare this derivation with the more customary one based on surface energies $[52,57,58]$. For the latter we assume the surface structure divides into a bulk solid covered with a well-defined quasi-liquid layer of thickness $d$. We write the surface energy as

$$
\begin{aligned}
\gamma(d) & =\gamma_{\mathrm{SL}}+\gamma_{\mathrm{LV}}+f(d)\left(\gamma_{\mathrm{SV}}-\gamma_{\mathrm{SL}}-\gamma_{\mathrm{LV}}\right) \\
& =\gamma_{\mathrm{SL}}+\gamma_{\mathrm{LV}}+f(d) \Delta \gamma,
\end{aligned}
$$

where $f(d)$ is as above, $\gamma_{\mathrm{SL}}$ is the energy of a solid-liquid interface, $\gamma_{\mathrm{LV}}$ is the energy of a liquid-vapour interface, and $\gamma_{\mathrm{SV}}$ is the energy of a fictitious 'dry' solid-vapour interface, for which there is no surface melting. The quantity $\gamma_{\mathrm{SV}}$ is in fact unmeasurable in the presence of surface melting, since then one cannot make a stable solid-vapour interface. If surface melting occurs, then $\gamma_{\mathrm{SV}}$ must be greater than $\gamma_{\mathrm{SL}}+\gamma_{\mathrm{LV}}$, so $\Delta \gamma$ is positive.

The free energy per unit area of such a surface, as a function of layer thickness, is given by

$$
F(d)=q_{\mathrm{m}} \rho t d+\gamma(d)
$$


where $q_{\mathrm{m}}$ is the latent heat of solidification per unit mass, $\rho$ is the water density, and again $t=\left(T_{\mathrm{m}}-T\right) / T_{\mathrm{m}}$. The first term in this expression reflects the free energy cost of melting the quasi-liquid layer below $T_{\mathrm{m}}$.

Minimizing the free energy as a function of $d$ yields

$$
f^{\prime}=-\frac{q_{\mathrm{m}} \rho t}{\Delta \gamma}
$$

and for $f(d)=\exp (-d / \lambda)$ we obtain

$$
d(T)=\lambda \log \left(\frac{\Delta \gamma}{q_{\mathrm{m}} \rho t \lambda}\right) .
$$

Although the two derivations are quite different, and use different input parameters, both give the same functional form for $d(T)$. Both are mainly useful at a qualitative level, since the input parameters $\Delta \kappa, \Delta \gamma, \lambda$ and $f(d)$ cannot yet be calculated or directly measured at an interesting level.

Many materials, although not all, exhibit surface melting to some degree near the melting point. The first speculations on the subject were made by Faraday in connection with some peculiar properties of ice, and many experiments have observed the effects of surface melting in ice [33]. The measurements generally indicate that $d(T)$ follows the logarithmic relation described above. There is, however, great disparity between the different measurements, probably because most experiments do not measure $d(T)$ absolutely. Rather they measure some quantity proportional to $d(T)$, with a poorly known proportionality constant (see $[59,60]$ and references therein for a review of recent measurements). Molecular dynamics simulations are making progress toward understanding the surface structure of ice, including surface melting [61-63], although reliable quantitative results are elusive. There also exists the possibility of kinetic surface melting, in an analogous sense to kinetic roughening, but to my knowledge this possibility has not been explored to any substantial degree.

Surface melting has a profound effect on the surface structure of ice, and attachment kinetics undoubtedly depends on surface structure. Thus we expect that surface melting must play an important role in the growth of snow crystals. Two additional facts support this: (1) surface melting is known to depend strongly on temperature over just the range where snow crystal growth shows a great deal of variation with temperature, and (2) different facets of the same solid sometimes exhibit the effects of surface melting differently. Thus it may well be that much of the temperature variation seen in the morphology diagram is a manifestation of surface melting in ice.

Unfortunately, at present we do not know, even qualitatively, how surface melting in general affects the attachment kinetics governing crystal growth [64]. The Lacmann-StranskiKuroda model [57,65] (for a summary, see [10] and references therein) is perhaps the most ambitious attempt to model crystal growth in the presence of surface melting, aimed specifically at understanding the snow crystal morphology diagram. The model is quite speculative, however, and we will see below that its main results do not agree with measurements of ice growth.

\subsection{Chemically enhanced growth}

The reader should come away from the above discussion with an indication that the surface structure of pure ice, even in equilibrium, is quite complex near the melting point and remains only poorly understood. This makes it especially difficult to understand the crystal growth of ice, since crystal growth is a dynamical phenomenon that likely depends strongly on the details of surface structure. As a result, our basic picture of ice crystal growth remains murky, 
and we do not yet have even a qualitative understanding of the underlying physics behind the snow crystal morphology diagram.

The whole story becomes vastly richer, however, when we consider how surface chemistry can affect the crystal growth process. There is much evidence that dilute chemical vapours in air can dramatically change the growth of ice crystals from water vapour [22,30]. A wide range of chemical impurities, such as vapours from many alcohols, acids, hydrocarbons, etc, even at low concentrations, affect both ice crystal morphologies and growth rates. This is one example of surfactant-mediated crystal growth, a topic that is being studied with increasing interest by the semiconductor community [66,67]. In the case of ice, very little has been quantified on this subject, and one can only speculate about how chemical catalysis affects the attachment kinetics behind ice crystal growth. To a large degree, the influence of surface chemistry on snow crystal growth remains a wide-open problem.

\section{Prism growth from vapour-observations}

Crystal growth theory is not sophisticated enough that one can calculate the formation of most real crystals from first principles, since the surface dynamics involved in growth can be quite complex. In general, there exists a large gap between crystal growth theory and the phenomenology of growing real crystals. This is especially true with a material like ice, for which even the equilibrium surface structure is not well known. Experiments, therefore, are very much needed to establish a self-consistent phenomenological picture of the growth process, which can then be used to guide further theoretical development.

In the case of ice, the simplest experiments are those involving the growth of hexagonal prisms, for which structural considerations are the least complex. If we assume the growth of an ice surface can be parameterized by the condensation coefficient $\alpha$, then a clear goal of experiments is to determine $\alpha\left(T, \sigma_{\text {surf }}\right)$ over as large a range of temperatures and supersaturations as possible. A substantial number of experiments have measured the growth of simple ice prisms with this goal in mind.

\subsection{Experimental techniques}

The first step in growing ice crystals is to produce a supersaturated environment, which can be done via several methods. One popular technique is to grow crystals on a substrate at some temperature $T_{1}$ while providing water vapour from a nearby ice reservoir at a higher temperature $T_{2}$. This method works especially well under near-vacuum conditions, and the supersaturation can be easily adjusted by changing $T_{2}$. One can also produce an isothermal supersaturated environment by providing a reservoir filled with a liquid salt solution, and then the supersaturation is varied by changing the salt concentration [68].

Another popular approach for providing an isothermal supersaturated environment is via a fog of supercooled liquid water droplets. Once such an artificial cloud has settled down, the supersaturation will be roughly equal to the water saturation value, $\sigma_{\text {water }}=\left(c_{\text {water }}-c_{\text {ice }}\right) / c_{\text {ice }}$. One could then vary $\sigma$ by using a fog of salty water droplets, but to my knowledge this approach has never been used. Clouds are most useful when experiments are done in air or other background gases at pressures near one atmosphere, although they have been used at higher pressures as well [69].

Vertical diffusion chambers $[8,21,30,70,71]$ are able to produce environments in which the supersaturation is quite high, easily up to $\sigma \approx 1$ or higher. The vertical temperature gradients in these chambers are also useful when investigating growth at different temperatures. Here again, diffusion chambers are mostly used in air or other background gases at pressures near 
one atmosphere, since the water vapour fluxes at significantly lower pressures would be quite large.

Once a supersaturated environment has been made, it is then necessary to nucleate some ice crystals. Ice will readily nucleate on most substrates, but only if the supersaturation is above some critical value that depends on the substrate composition [72]. In some circumstances it is useful to introduce a brief pulse of water vapour to nucleate crystals on a substrate. The nucleated crystals then quickly consume the excess water vapour in the pulse, leaving small crystals to grow at lower supersaturations [73].

Some additional popular methods to nucleate ice crystals are: (1) introducing a small quantity of silver iodide smoke, since the smoke particles serve as good nucleation sites, (2) rapidly expanding a small quantity of saturated gas to bring about homogeneous nucleation [74], and (3) dropping a particle of dry ice into a growth chamber, which again induces homogeneous nucleation in the cold wake of the particle. These techniques are often used to produce free-falling crystals in an air-filled cold chamber.

Ice will grow epitaxially on the mineral covellite $(\mathrm{CuS})$ [75], on silver iodide crystals $[71,72,75]$, and on mica [76], to name a few. In some instances the nucleation on silver iodide is optically deactivated [77]. For all these minerals, ice crystals grow with a basal plane in contact with the substrate, and interference effects can be employed to determine the crystal thickness [78]. To my knowledge, the controlled epitaxial growth of ice crystals with other crystal orientations has not been realized.

Some new experimental developments include the observation of levitated single ice crystals, both in a moving air column [79] and via electrodynamic levitation [80]. The obvious advantage of such techniques is that one can monitor the growth of a single, isolated crystal as a function of time. Determining $\sigma$ accurately is still a difficult problem with these experiments, however.

Negative crystals (vapour inclusions) can also be used in ice evaporation studies, which are related to growth studies. But in experiments with negative crystals it is especially difficult to accurately determine the subsaturation at the crystal surface, which hinders quantitative experiments $[81,82]$.

3.1.1. Systematic errors in ice crystal growth measurements. At least one thing has become abundantly clear over several decades of ice crystal growth experiments - there are a great many troublesome systematic effects that can influence ice crystal growth and thwart one's attempts to make quantitative measurements under well-controlled conditions. If the experimenter is not exceedingly careful, a measurement may be dominated by systematic effects. Thus it is worthwhile to examine these various problems in detail, so that one can avoid them in future experiments. The list here is a summary; a more detailed account of systematic problems present in published experiments can be found in [83].

Vapour diffusion. Unless an ice crystal growth experiment is done under pure water vapour conditions - that is, in the complete absence of any background gas-one must worry about vapour diffusion effects. Diffusion reduces $\sigma_{\text {surf }}$ relative to $\sigma_{\infty}$ and thus limits crystal growth. Equation (4) above shows that it is nearly impossible to make an accurate measurement of $\alpha$ when $\alpha_{\text {diff }}<\alpha$. Of course one can, and should, model the effects of diffusion to determine $\sigma_{\text {surf }}$ for growing crystals, but this can only be done with limited precision. If $\alpha_{\text {diff }}$ is small, then even modest systematic errors in a crystal growth measurement will be amplified into large systematic errors in the determination of $\alpha$. The very best quantitative ice crystal growth experiments done to date are only absolutely accurate to about a factor of two in the measured growth velocity, with crystal-to-crystal variations that are again roughly a factor of two. This means one 
cannot make a meaningful measurement of $\alpha$ unless $\alpha_{\text {diff }} \gtrsim \alpha$. This is an important point, since nearly all the early ice growth experiments, particularly those done in air, did not adequately disentangle diffusion effects from the growth data, and thus produced unreliable results [83].

Neighbouring crystals. Systematic errors from diffusion can be present even when $\alpha_{\text {diff }}>\alpha$, if the crystal under observation is surrounded by neighbouring crystals. A notable example is the case where one is observing the slow growth of a faceted crystal when it is surrounded by fastgrowing, nonfaceted crystals. Nonfaceted ice surfaces can have $\alpha \approx 1$, meaning that $\sigma \rightarrow 0$ in their vicinity, which can greatly impede the growth of nearby faceted crystals. The best way to see this is by considering the scalar field $\sigma(x)$ in the space around the growing crystals. Diffusion requires $\nabla^{2} \sigma=0$, and the fast-growing nonfaceted crystals may be modelled by boundary conditions with $\sigma \approx 0$ at the growing surfaces. If the water vapour source is far away, then the nonfaceted crystals may substantially reduce $\sigma$ near the crystal under observation. This is not the case in a pure water vapour environment, but is a concern with surprisingly low background gas pressures. The solution to this problem is to (1) make sure there are not too many neighbour crystals, (2) make sure the neighbour crystals are far away from the crystal under observation, and (3) keep the water vapour source close.

Crystal heating. The simple spherical analysis shows how crystal heating affects growth measurements. If latent heat is carried away by conduction to the background gas, then equation (12) shows that heating is important when $\alpha_{\text {cond }}<\alpha$. A substrate is more effective at removing heat, and in this case equation (16) shows that heating is important when $\alpha_{\text {cond }}^{\prime}<\alpha$. Heating is especially problematic for crystals not in contact with a substrate, and for growth at low background gas pressures. Again this systematic error is reduced by making measurements using smaller crystals.

Cloud dynamics. When ice crystals are growing in a cloud of water droplets, it is a mistake to assume that the supersaturation is automatically equal to the equilibrium value for liquid water at the cloud temperature. It takes time for a cloud to reach a state of near equilibrium, and how close one approaches equilibrium depends on the cloud size and dynamics. Large droplets may be warmer than ambient if they have not had time to equilibrate. Small droplets may be too cool if evaporative cooling draws heat out faster than interaction with the surroundings can replace it. In addition, when many crystals are growing in a cloud they all compete for water vapour, again distorting the supersaturation field. These effects should be carefully modelled in a given experiment to gain a more accurate estimate of $\sigma$ inside the cloud. Note that these problems are much worse for small clouds and when the air pressure is reduced.

Crystal imperfections. There is ample evidence that crystal imperfections in faceted ice surfaces can serve as a source of molecular steps that promote growth. Screw dislocations are well known in this role, but other crystal faults may also be relevant. A ready source of molecular steps will especially promote growth at low supersaturations, when two-dimensional nucleation is rare. My experience (following [84]) has been that imperfect surfaces often cannot be visually distinguished from more perfect faceted surfaces; some crystal specimens simply exhibit anomalously fast growth. The best experimental strategy here is to measure a large number of crystals.

Substrate interactions. Several papers have described how substrate interactions can affect the growth of ice crystal facets $[73,85,86]$. It appears that the intersection of an ice facet with a substrate can be a source of molecular steps, which again increases the growth rate 
when the growth is nucleation-limited [85]. These effects are probably not always important, and different substrates may behave differently. One thing is clear, however-substrate interactions can increase ice growth rates by at least factors of $2-5$, so this systematic effect must be considered. The best approach is to restrict one's measurements to facets that are not in contact with a substrate [39].

Chemical impurities. Impurities on an ice surface might impede growth by interfering with surface diffusion, or they might enhance growth by providing nucleation sites. At any rate, there is certainly ample qualitative evidence that impurities affect ice growth $[22,30]$. This is an especially thorny experimental problem, since there is little quantitative data on how clean a given experiment must be before it can be considered free of chemical effects. However, crystals grown in normal laboratory air repeatably exhibit morphological changes with temperature that follow the morphology diagram, so we can conclude that the impurities in ordinary air do not disrupt the growth of new small crystals by a large factor. My personal rule-of-thumb is that if you can smell something in the air, it will likely have some effect on ice crystal growth.

Evaporation/growth cycling. There is some evidence [87] that evaporating and regrowing a single crystal affects the measured growth rates, probably from the introduction of nucleation sites as the crystal is processed. The best experimental strategy here is to avoid a lot of evaporation/growing cycles when taking growth data, and to sample many crystals.

In summary, past experience shows that there are many pitfalls that must be avoided when measuring ice crystal growth rates. These systematic effects should be examined carefully when designing new experiments, and they should be examined again when interpreting the results of published experiments.

\subsection{Conclusions from growth measurements}

Considerable effort has gone into making quantitative measurements of the growth of simple ice prisms and/or facet surfaces in order to accurately determine $\alpha\left(T, \sigma_{\text {surf }}\right)$ and thus gain a better understanding of the snow crystal morphology diagram. The measurements have proven to be surprisingly difficult, however, and a critical examination of the literature reveals that essentially all the results published to date suffered from systematic errors to some degree (for a detailed summary of the experiments see [83]). As a result, the different experiments are not in reasonable agreement with one another, and we must therefore be cautious in what we conclude from the data. With that in mind, we have attempted here to summarize what is known, or at least probably known, about the growth of simple ice prisms. Some of these conclusions reflect the biases of the author and are clearly in need of additional experimental and/or theoretical study.

The parameterization in terms of a condensation coefficient is a valid one. This is a weak conclusion, since the existing growth data neither support it strongly nor suggest that it is invalid. The measurements roughly indicate $\alpha<1$ on faceted surfaces and $\alpha \approx 1$ on rough surfaces, at least to the limited accuracy of the most reliable data [27,39]. Thus it is certainly useful to assume $\alpha\left(T, \sigma_{\text {surf }}\right)$ is a valid parameterization for simple ice facets. However, there is also evidence (see below) that $\alpha$ depends on the size and geometry of a given facet, and not just on $T$ and $\sigma_{\text {surf }}$, which complicates the picture substantially.

Substantial surface diffusion between facets seems unlikely. Here again this is a weak conclusion, without a great deal of hard experimental evidence one way or the other. Mason 
and collaborators have suggested that surface diffusion between facets is important [88], and this result has been widely cited. But it appears the data in support of this were substantially distorted by diffusion effects [83]. On the theoretical side, there is a potential barrier that inhibits the migration of admolecules between terraces (the Schwoebel-Ehrlich effect [34,36]), and surface diffusion between facets would require numerous such jumps to traverse a rounded corner. Without compelling experimental evidence demonstrating surface diffusion between facets, it seems prudent at this stage to assume the phenomenon is unlikely. Ice is full of surprises, however, and the data are certainly not such that we can rule this possibility out.

The condensation coefficient is independent of background air pressure. Here again, several authors have presented data suggesting that background gas does affect $\alpha\left(T, \sigma_{\text {surf }}\right)$, but upon close inspection these data were almost certainly influenced by diffusion effects, and in my opinion they are not reliable [83]. The molecular interactions between air and ice are sufficiently weak that it seems unlikely that the presence of air would have a significant influence on the molecular dynamics of the ice surface. There is also evidence that snow crystal morphologies are essentially the same in gases of air, nitrogen, helium, hydrogen and carbon dioxide, even though these gases have much different solubilities in water, reflecting different strengths of molecular interactions [89]. Here again, we cannot rule out that $\alpha$ changes to some degree with background gas, and better data are needed. But in my opinion it appears that $\alpha$ is essentially independent of background gas, at least for relatively inert gases like air.

Facet growth is primarily nucleation-limited over a substantial temperature range. This conclusion is based largely on recent data [39], which are the most thorough and appear to be the least affected by systematic errors (however, see [83]). Thus it strictly applies only in the temperature range $-40^{\circ} \mathrm{C}<T<-10^{\circ} \mathrm{C}$, for the growth of facets that do not include step sources from crystal faults or impurities. The data over this entire temperature range are well represented by a condensation coefficient with the functional form $\alpha\left(T, \sigma_{\text {surf }}\right)=A(T) \exp \left(-\sigma_{\text {crit }} / \sigma_{\text {surf }}\right)$, which is consistent with nucleation-limited growth. Preliminary new data [90] suggest that this functional form continues to represent the data adequately at temperatures up to $T=-5^{\circ} \mathrm{C}$.

The formation of six-fold symmetric snow crystal prisms is a direct indication that the growth rates of the six prism facets on a given crystal are all comparable. This immediately suggests that the growth of prism facets under typical meteorological conditions is often not driven by dislocations, since it is unlikely that the dislocation density would be the same on all six facets of a symmetrical crystal. This supports our conclusion that growth is primarily nucleation-limited.

In contrast, observations of Antarctic snow crystals reveal a large fraction of asymmetric prisms [91], suggesting the growth of those crystals is likely driven by dislocations. Antarctic crystals often grow at low temperatures under conditions where $\sigma_{\text {surf }} \ll \sigma_{\text {crit }}$, so again this fits our overall picture.

The critical supersaturation is roughly the same for prism and basal facets, and is a monotonic function of temperature over a substantial temperature range. It was speculated for many years that large differences in $\sigma_{\text {crit }}$ between the basal and prism facets, together with changes in $\sigma_{\text {crit }}$ with temperature, would be responsible for much of what is seen in the morphology diagram. Surprisingly, it now appears that $\sigma_{\text {crit }}$ is roughly the same for both the basal and prism facets, and furthermore changes only monotonically with temperature. This conclusion is again based largely on the most recent data [39], which are shown in figure 6. Other data sets give conflicting results, but the recent data suffer the least from systematic errors [83]. Additional confirmation is certainly desirable, as is an extension of these data to higher temperatures. 

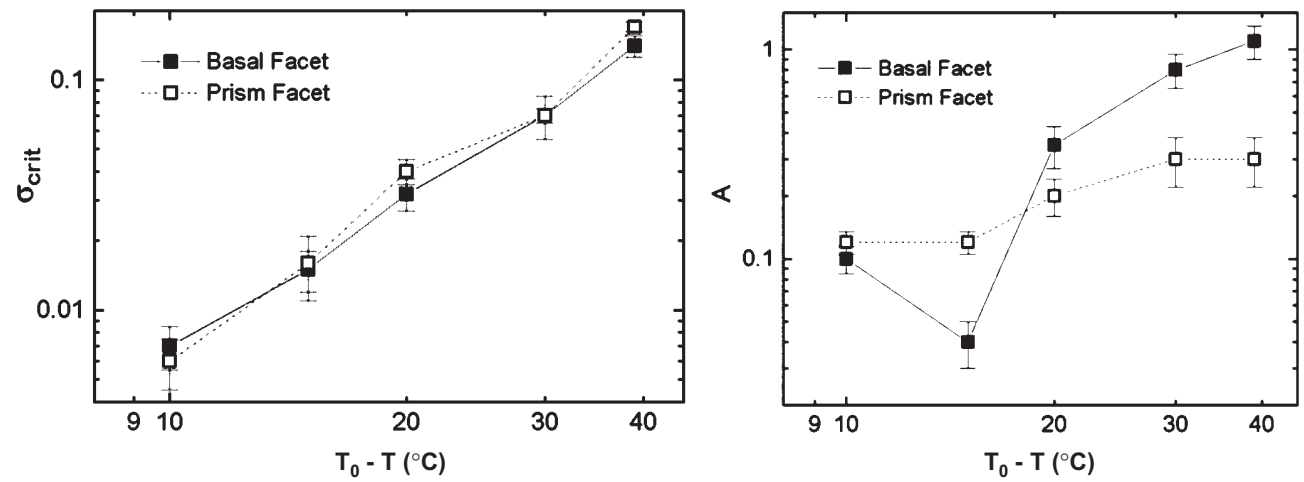

Figure 6. Results from measurements of the growth of simple ice prisms from the vapour phase. The growth was parameterized in terms of a condensation coefficient with $\alpha\left(T, \sigma_{\text {surf }}\right)=$ $A \exp \left(-\sigma_{\text {crit }} / \sigma_{\text {surf }}\right)$. The plots show $\sigma_{\text {crit }}(T)$ and $A(T)$ that were extracted from the data in [39]. Note that the critical supersaturation $\sigma_{\text {crit }}$ is essentially identical for the prism and basal facets, with a relatively simple, monotonic dependence on temperature. The constant term $A$, on the other hand, is different for the two facets with a more complex temperature dependence.

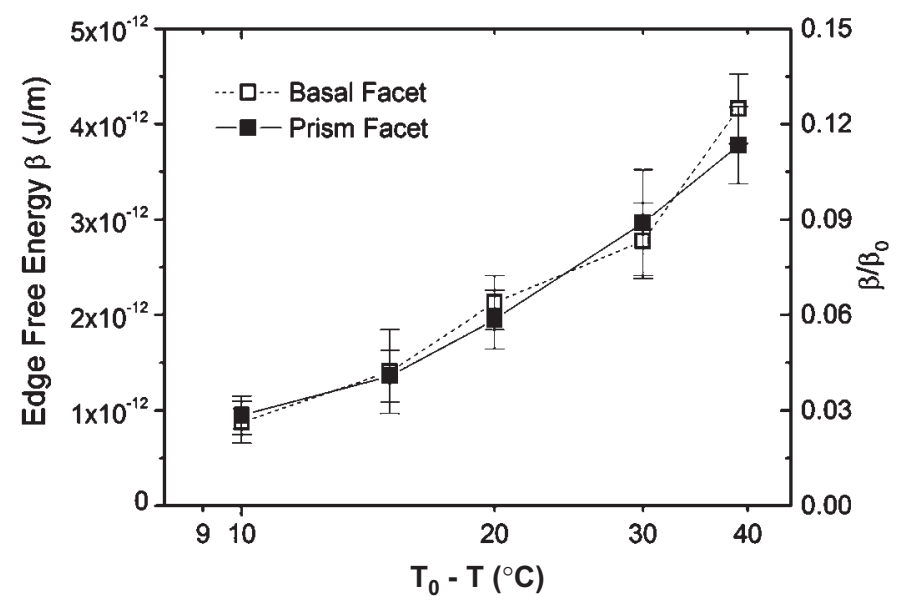

Figure 7. The edge free energy, $\beta$ (per unit length) for a growth island that is one molecular layer thick, as a function of temperature [39]. The scale at right is normalized by $\beta_{0}=a \gamma$, where $a$ is the layer height and $\gamma$ is the surface energy (per unit area) for ice.

A measurement of the critical supersaturation then gives $\beta$, the edge free energy for a growth island, according to equation (20), and this is shown in figure 7. The diminishing edge free energy with increasing temperature is probably indicative of surface restructuring; at higher temperatures the lattice structure becomes less rigid, allowing the step edge to be smoother with a lower edge free energy. This could be confirmed by molecular dynamics simulations, which appears to be feasible with current technology.

A roughening transition occurs on prism facets near the melting point. This appears to occur at a temperature of $T \approx-2^{\circ} \mathrm{C}$, and only on the prism facets; the basal facets do not show a similar transition below the melting point [38]. Snow crystals sometimes grow as plate-like forms with no prism facets at these warm temperatures, supporting this conclusion. Kinetic roughening may also be important at these temperatures. Since surface melting is almost 
certainly present this close to the melting point, the roughening transition must be at the quasiliquid/solid interface. Observations of ice growth from liquid water also show basal faceting without prism faceting [92-94], which likely indicates the same basic roughening transition.

Prism growth in air follows the morphology diagram. Measurements of the sizes of prism crystals grown in air at a pressure near 1 atm clearly follow the morphology diagram [10,25]. The prism sizes follow a power-law behaviour as a function of growth time, as expected when the growth is primarily diffusion limited [26,27]. The aspect ratios of such prisms commonly range from $D / T \approx 40$ (diameter/thickness) for thin plates grown at $T=-15^{\circ} \mathrm{C}$ to $D / T \approx 1 / 20$ for columnar prisms grown near $T=-5^{\circ} \mathrm{C}$.

Ice prisms grow nearly isometrically in near-vacuum at all temperatures. In contrast to the extreme aspect ratios seen in prisms grown in air, ice prisms grown in near-vacuum conditions typically have $1 / 3<D / T<3$, regardless of temperature. This is a robust result that has been found in several experiments, provided there are no strong ice/substrate interactions. As the background pressure is increased the aspect ratios become more extreme, and the morphological structure becomes more complex [69]. The nature of the transition to more extreme aspect ratios as pressure is increased is certainly deserving of additional study.

A new type of instability is needed to explain the growth of thin plates and columns. The growth of nearly isometric crystals in near-vacuum is difficult to reconcile with the observation that thin plates and needles often grow in air. Diffusion actually tends to discourage the growth of ice prisms with high aspect ratios, because in such cases the supersaturation is lowest on the fast-growing surfaces [27]. For the growth of thin plates at $T=-15^{\circ} \mathrm{C}$, for example, we see from figure 5 that $\alpha_{\text {prism }} / \alpha_{\text {basal }}>v_{\text {prism }} / v_{\text {basal }}$, and under some circumstances we must have $\alpha_{\text {prism }} / \alpha_{\text {basal }} \approx 100$ to produce the types of plates shown in figure 5 [27]. This conclusion follows rather simply from our discussion of the diffusion equation above. Under near-vacuum conditions, however, the growth is more nearly isometric, indicating that $\alpha_{\text {prism }} / \alpha_{\text {basal }} \ll 100$ in that case.

To resolve this puzzle, Libbrecht has suggested that a new type of growth instability is necessary to reconcile these various observational facts [27]. He suggested that $\alpha$ may change not only with temperature and supersaturation, but also with the size of the crystal facet, such that $\alpha \rightarrow 1$ when the facet size becomes small, calling this structure-dependent attachment kinetics. This is a speculative model, without a strong theoretical foundation, so additional work is needed to understand this aspect of the growth dynamics. However, it does appear that a simple $\alpha(\sigma, T)$ function cannot explain the growth of ice crystals in air and in near-vacuum, and some form of new instability is necessary to reconcile these data [27]. The true physical nature of this instability remains a mystery.

\subsection{Summary of snow crystal prism growth}

One might have thought that understanding the growth of simple snow crystal prisms, the most basic ice crystal form, would be a relatively simple task. Upon closer inspection, however, the problem is surprisingly rich, and many aspects remain quite puzzling. Although considerable effort has been expended in attempts to reveal the physical origins of the morphology diagram, at present we do not have even a satisfactory qualitative picture of the mechanisms that produce such dramatic changes in morphology with temperature.

Our understanding of ice prism growth continues to fall short in both theory and experiment. On the theoretical side, we do not yet have a microscopic model that describes 
crystal growth from the vapour in the presence of surface melting. There is a general expectation that surface melting is an important piece of the ice prism puzzle, but a quantitative growth model remains elusive. The Lacmann-Stranski-Kuroda model [57, 65] (for a summary, see [10] and references therein) predicts large nonmonotonic changes in $\sigma_{\text {crit }}$ with temperature and large differences in $\sigma_{\text {crit }}$ between the prism and basal facets, and both predictions are contradicted by experiment.

In addition to this lack of a basic underlying growth model, there is now evidence that additional growth instabilities, such as the proposed structure-dependent attachment kinetics, are necessary to explain some aspects of prism growth, as described above. These new instabilities are likely quite important in understanding the morphology diagram, but they also complicate the theoretical picture even further.

On the experimental side, many measurements of $\alpha\left(T, \sigma_{\text {surf }}\right)$ have been seriously affected by systematic errors [83], so we still do not have a good picture of $\alpha\left(T, \sigma_{\text {surf }}\right)$ over a broad range of temperatures and supersaturations. As a result, we have not yet mapped out the ice attachment kinetics in detail, and thus we are missing this vital input to the growth problem. This situation is changing, however, as new experiments reduce the systematic effects and produce more reliable measurements. We are confident that substantial experimental progress will be made in this area, and that this will lead to new theoretical insights into the basic microscopic processes governing snow crystal growth.

\section{Dendritic growth}

The simple hexagonal ice prisms discussed above appear when the crystal growth is not so strongly limited by particle diffusion - that is, when the supersaturation is low, the crystal size is small, and/or the background gas pressure is low. As long as at least one of these parameters is small enough, the growth is dominated by attachment kinetics, resulting in faceted crystals. When some combination of these parameters is increased sufficiently, then particle diffusion begins to dominate the crystal growth dynamics and at some point branching occurs. When the crystal morphology exhibits numerous branches and side-branches, then we say the growth is dendritic, which literally means 'tree-like'. Dendritic crystal growth is but one example of the more general phenomenon of pattern formation in nonequilibrium growing systems [95, 96].

Some examples of ice crystal dendrites grown in air are shown in figure 8. While ice dendrites can grow at any temperature, they are most prominent near $T=-5^{\circ} \mathrm{C}$, where thin ice needles form, and especially near $T=-15^{\circ} \mathrm{C}$, where the six-fold crystal symmetry produces side-branches that extend at $60^{\circ}$ angles from their main branches. In both these cases, the growth of a single ice dendrite is largely two-dimensional, such that most of the large-scale structure is confined to a flat plane. The overall complexity of the dendritic pattern increases with increasing supersaturation, as indicated in the morphology diagram (figure 2), as well as with increasing crystal size and with increasing background gas density [69]. As is typical for dendrites, the growth often exhibits some self-similar, fractal characteristics. For ice dendrites the smallest structures typically occur on scales of about $1-10 \mu \mathrm{m}$.

\subsection{The transition from faceting to branching}

Whenever solidification is limited by diffusion, then the growth of a flat interface is unstable. This is true whether the growth is limited by particle diffusion that brings material to the interface or by heat diffusion that removes latent heat generated at the interface. To see the origin of this instability, consider the case where particle diffusion is the dominant factor and ignore attachment kinetics. If we start with a flat, growing surface and add a small bump, the 


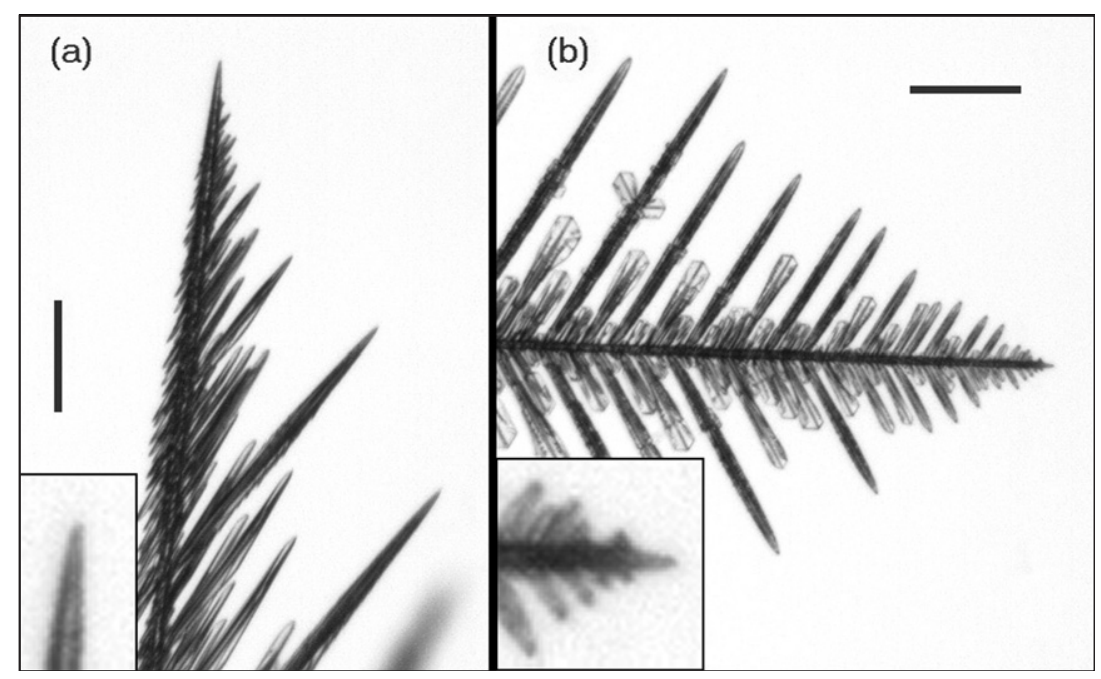

Figure 8. (a) An ice dendrite growing at $T=-5^{\circ} \mathrm{C}$, showing the characteristic 'fishbone' structure. The tip growth is not along a well-defined crystal axis, but varies depending on supersaturation. Most of the structure seen here is confined to a flat plane. (b) A 'fern-like' ice dendrite growing at $T=-15^{\circ} \mathrm{C}$. Here the tip growth is along the $a$-axis of the crystal, so each side-branch makes a $60^{\circ}$ angle from its main branch. Growth of the basal facet is slow at $-15^{\circ} \mathrm{C}$, so again this is a planar structure. In both panels the scale bars are $100 \mu \mathrm{m}$ long and the insets are $4 \times$ magnified views of the growing tips. While growing, the dendrite tip velocity $v_{\text {tip }}$ and the tip radius of curvature $R_{\text {tip }}$ are approximately constant. These crystals were grown in air at a pressure of $1 \mathrm{~atm}$ at $\sigma_{\infty} \approx 1$, where typical tip velocities are a few micrometres per second and typical tip radii are of order $1 \mu \mathrm{m}$ (from [97]).

bump will effectively stick out farther into the supersaturated medium above the surface. Thus particle diffusion will bring material to the top of the bump at a slightly faster rate than to the surrounding flat surface. With a greater supply of material, the bump grows larger, which increases its supply even more. This positive feedback effect is often called the MullinsSekerka instability [34, 98,99], and it is a ubiquitous feature of diffusion-limited growth. Much of the structure in snow crystals ultimately derives from this instability.

If we add attachment kinetics at the interface, the picture becomes more complex. We then find that a simple faceted morphology can grow stably, but only for so long. Consider the case shown in figure 9, where a faceted plate-like crystal sprouts branches as it grows larger. If we focus on the growth of the prism facets, then the growth of a flat facet requires that $v(x) \approx$ constant, where this is the perpendicular growth velocity as a function of position along the facet. From kinetics we have $v=\alpha v_{\text {kin }} \sigma_{\text {surf }}$, and a solution of the diffusion equation reveals that $\sigma_{\text {surf }}(x)$ varies across the facets, being lowest at the facet centres. Thus as long as the crystal retains its simple prism structure as it grows, $\alpha$ must also vary across the facets, being highest at the facet centres. Since the surface diffusion lengths are small compared to the crystal size, this simple analysis reveals that the prism facets cannot be perfectly flat, but are slightly concave. The curvature adjusts itself so that the step density is higher at the facet centres, which provides the necessary variation in $\alpha(x)$ across the facet. The curvature is so slight, however, that the facets remain flat in appearance.

As long as this growth feedback mechanism can continue, the crystal growth remains faceted in appearance, with the facet curvature increasing steadily as the crystal grows larger. With time the step density reaches its maximum value at the facet centres, at which point the surface is essentially rough on a molecular scale. Then $\alpha \approx 1$, its maximum value, while $\sigma_{\text {surf }}$ 


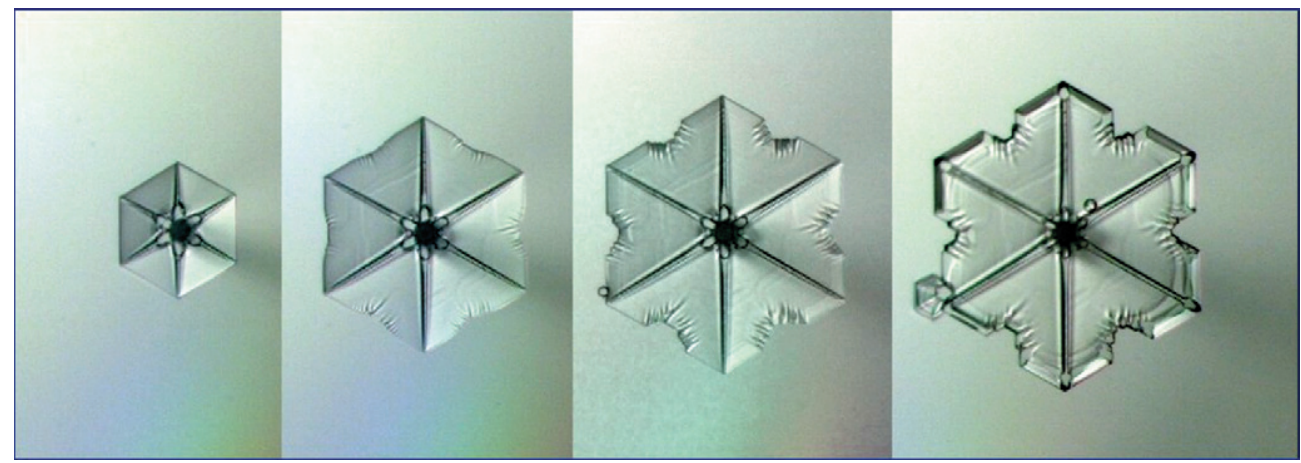

Figure 9. A series of photographs of a growing snow crystal, showing the transition from faceted to branched growth. The initially flat prism facets become hollowed as particle diffusion limits the growth at the facet centres. This plate-like crystal was grown on the end of an electric needle (see below), in air at a pressure of $1 \mathrm{~atm}$. The final crystal size is approximately $1 \mathrm{~mm}$. Note that a small water droplet fell on the lower left tip of the crystal as it grew, nucleating the growth of a secondary structure. (Photo by the author.)

continues to decline at the facet centres as the crystal continues to grow. Once this condition is reached, we can no longer have $v(x) \approx$ constant across the facet, and the crystal then sprouts branches as shown in figure 9.

Near $T=-15^{\circ} \mathrm{C}$ the Mullins-Sekerka instability leads to the familiar six-fold-symmetric branching seen in plate-like stellar crystals. Near $T=-5^{\circ} \mathrm{C}$ the same instability first produces hollow columns and then clusters of needle crystals. Note that convex facets do not undergo this branching instability. Thus the growth at $T=-15^{\circ} \mathrm{C}$ remains plate-like even at high supersaturations and for large crystal sizes.

The transition from the growth of simple prism crystals to more complex dendritic structures is fairly well understood at a qualitative level, and some attempts have been made to model this transition in detail [43]. To my knowledge, however, little quantitative experimental work has been done to examine this transition. The picture is particularly complicated in ice, since faceting continues to play a strong role even in the presence of well-developed branching. For example, the fully developed dendritic growth at $T=-15^{\circ} \mathrm{C}$ (see figure $8(b)$ ) still retains strong faceting on the basal surfaces, yielding a nearly two-dimensional dendritic structure. Faceting is also frequently seen at the tips of branches, as in figure 9.

\subsection{Dendrite theory and snow crystal growth}

In spite of the overall complexity of dendritic growth, the tip of a dendrite often grows with a tip velocity $v_{\text {tip }}$ and a tip radius of curvature $R_{\text {tip }}$ that do not change appreciably with time (see figure 8). This statement is true for many different types of diffusion-limited solidification, for structures growing from the vapour or the melt phase, regardless of whether the growth is limited by particle diffusion or heat diffusion [98]. Many of the essential features of dendrite growth theory have been worked out in considerable detail [100,101], and this theory can be fairly easily adapted to ice crystal growth from the vapour phase [97]. The theory is not complete in that it does not include faceting and other complications, so at present it cannot quantitatively describe many of the features seen in ice dendrites. Nevertheless, it is a good starting point that explains many aspects of ice dendrite growth to a reasonable approximation.

Dendrite theory in the case of snow crystal growth begins with the particle diffusion equation in the limit of small Peclet number, as was described above. In this limit the 
time-dependent diffusion equation is replaced by Laplace's equation $\nabla^{2} c=0$, which must then be solved for the water vapour concentration $c(x)$ with the appropriate boundary conditions. One can show that particle diffusion typically restricts growth more than heat diffusion for the case of snow crystal dendrites grown in air [97], so heat diffusion will be ignored in the following treatment.

The contribution of surface energy to the surface boundary condition can be found by considering a spherical crystal, which has the surface energy $U_{\text {surface }}=4 \pi R^{2} \gamma$, where $R$ is the radius of curvature and $\gamma$ is the surface tension, equal to $0.109 \mathrm{~J} \mathrm{~m}^{-2}$ for ice. Pulling a molecule off the surface reduces the sphere's surface area and thus releases an energy $\mathrm{d} U_{\text {surface }}=2 \gamma / c_{\text {solid }} R$. Therefore, the equilibrium vapour pressure above a spherical crystal is approximately

$$
c_{\mathrm{eq}} \approx c_{\mathrm{sat}}\left(1+\frac{2 \delta}{R}\right)
$$

where $\delta=\gamma / c_{\text {solid }} k T \approx 1 \mathrm{~nm}$ and $c_{\text {sat }}$ is the saturated vapour pressure above a flat interface.

Attachment kinetics also affects the surface boundary condition because a growing surface is not in equilibrium. From the definition of $\alpha$ we have

$$
v_{\mathrm{n}}=\alpha v_{\mathrm{kin}} \frac{\left(c_{\mathrm{surf}}-c_{\mathrm{eq}}\right)}{c_{\mathrm{eq}}}
$$

so to lowest order

$$
c_{\text {surf }} \approx c_{\text {sat }}\left(1+\frac{2 \delta}{R}+\frac{v_{\mathrm{n}}}{\alpha v_{\text {kin }}}\right) .
$$

This expression is strictly valid only for spherical growth in the case of isotropic surface tension and attachment kinetics, but we will proceed using this expression nevertheless, with the understanding that the more general case would be similar in form.

We note at this point that our goal in this section is to describe the essential physics of ice dendrite growth from the vapour, and not to find a mathematically precise solution to the full problem. Thus we will make numerous rather crude approximations when dealing with the dendrite geometry and the surface boundary conditions. This approach is instructive and quite productive, and it is also an unfortunate necessity because a full dendrite growth theory has not yet been derived. In particular, the combination of faceting and branching has proven to be a formidable problem. A much more mathematical treatment of the two-dimensional problem with attachment kinetics is described in [101], which reveals some of the full complexity inherent in dendrite growth.

We have found that a simple theoretical treatment of the problem works surprisingly well at a semi-quantitative level, apparently because both the phenomenon and the theory are quite robust and insensitive to perturbations. For example, the phenomenon is robust in that dendrite growth with time-independent $v_{\text {tip }}$ and $R_{\text {tip }}$ is a fairly ubiquitous occurrence over a broad range of physical circumstances. Small changes in attachment kinetics, geometry, etc, do not seem to alter the underlying physics that describes the growth. The basic results from dendrite theory also appear to be quite robust, and the theory even shows many similar features in both two-dimensional and three-dimensional formulations [102]. This latter property is probably why we can use the simple theory to such good effect when describing the growth of these flat-and thus strongly faceted-ice structures.

To solve the diffusion equation around a growing ice dendrite, we assume that the crystal growth proceeds with a typical three-dimensional dendritic morphology, described by an approximately cylindrically symmetric parabolic dendrite tip moving with constant $v_{\text {tip }}$ and $R_{\text {tip }}$. Typically we do observe that ice crystal dendrites have rather rounded tip structures, as 
seen in figure 8, suggesting that faceting is not well developed near the tip, where the theory is being applied. It is well known that such a parabolic form, with constant $v_{\text {tip }}$ and $R_{\text {tip }}$, is a steady-state solution to the diffusion equation in three-dimensional, called the Ivantsov solution [34]. In the limit of low Peclet number we are considering here, the Ivantsov solution can be written as (dropping the subscripts on $v_{\text {tip }}$ and $R_{\text {tip }}$ ) $[34,103]$

$$
v \approx \frac{2 D}{R \log \left(\eta_{\infty} / R\right)} \frac{c_{\mathrm{sat}}}{c_{\mathrm{solid}}}\left[\sigma_{\infty}-\frac{2 \delta}{R}-\frac{v}{\alpha v_{\mathrm{kin}}}\right],
$$

where $\sigma_{\infty}=\left(c_{\infty}-c_{\text {sat }}\right) / c_{\text {sat }}$ and $\eta_{\infty}$ is the parabolic coordinate of the far-away boundary of the system, at which point we have $c=c_{\infty}$. The log term in this expression appears because we are in a regime where the diffusion length is larger than the boundaries of the experimental apparatus, i.e. $\ell=2 D / v \gg \eta_{\infty}$. It is sufficient in most experimental cases to assume a constant $B \equiv \log \left(\eta_{\infty} / R\right) \approx 10$ [97]. This is consistent with our expectation that the particular details of the far-away boundary condition in the problem should not contribute greatly to the final dendrite solution.

When the growth is mainly diffusion limited we have $v \ll \alpha v_{\text {kin }}$, and we have found that for dendrite growth from vapour the capillary perturbation is typically negligible in comparison to the kinetic perturbation. (The opposite is true for growth from the melt, which is much more commonly discussed in the literature on diffusion-limited growth [101].) Thus we can substitute the unperturbed Ivantsov solution in for $v$ on the right-hand side of equation (24), which then gives to lowest order

$$
\begin{aligned}
v & \approx \frac{2 D}{B R} \frac{c_{\text {sat }}}{c_{\text {solid }}}\left[\sigma_{\infty}-\frac{R_{\mathrm{kin}}^{*}}{R}\right] \\
& =\frac{2 D}{B R} \frac{c_{\text {sat }}}{c_{\text {solid }}}\left[1-\frac{R_{\text {kin }}}{\alpha R}\right] \sigma_{\infty},
\end{aligned}
$$

where

$$
\begin{aligned}
R_{\mathrm{kin}}^{*} & =\frac{\sigma_{\infty}}{\alpha} \frac{2 D}{B} \sqrt{\frac{2 \pi m}{k T}} \\
& =\frac{\sigma_{\infty}}{\alpha} R_{\mathrm{kin}},
\end{aligned}
$$

which defines the terms $R_{\text {kin }}$ and $R_{\text {kin }}^{*}$ [97]. For ice growth in air at a pressure of 1 atm we have $R_{\text {kin }} \approx 30 \mathrm{~nm}$.

Note that the Ivantsov solution, equation (25) in our case, gives only a single relation between the tip velocity $v$ and radius $R$, here with small perturbations from the capillary and kinetic terms (assuming $\delta \ll R$ and $v \ll \alpha v_{\text {kin }}$ ). It is generally true for dendrite growth that the diffusion equation alone only provides a partial solution to the problem. An additional relation between these variables is required to select the correct $v$ and $R$.

A principal result from dendrite theory $[100,101]$ is that we can write down a dimensionless 'solvability' parameter that in our case becomes

$$
s=\frac{2 R_{\mathrm{kin}} D}{v R^{2}} \frac{\sigma_{\infty}}{\alpha} \frac{c_{\mathrm{sat}}}{c_{\text {solid }}}
$$

and the theory further dictates that $s$ will depend only on the intrinsic material properties, in this case mainly on the detailed properties of the attachment kinetics. Thus $s$ will be essentially a constant term, with no direct dependence on $D, \sigma_{\infty}$, or other extrinsic variables.

To my knowledge there is no simple derivation of this seemingly simple result. As has been noted in the context of solvability theory (e.g. [100]), equation (29) follows from the fact that attachment kinetics here provides the additional length scale $R_{\text {kin }}^{*}$ necessary for uniquely 

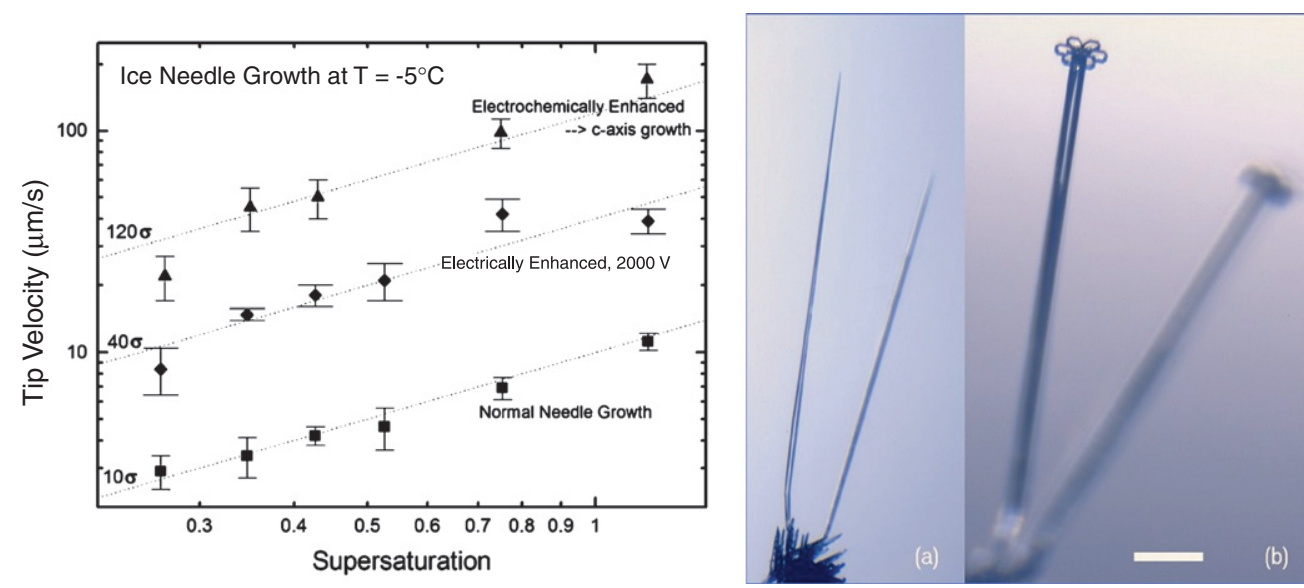

Figure 10. Left: Measurements of ice needle growth at $T=-5^{\circ} \mathrm{C}$ as a function of supersaturation, $\sigma$, for normal growth (lower points), electrically enhanced growth (middle points), and electrochemically enhanced growth (upper points). Right: Photographs of $c$-axis electric needles grown at $T=-5^{\circ} \mathrm{C}$ under the influence of chemical additives. The picture in $(a)$ shows the electric growth phase. In $(b)$ the field had been removed and the temperature changed to $T=-15^{\circ} \mathrm{C}$, allowing normal growth to take place. Then plate-like crystals grew on the needle tips. Data and photos from [97].

determining the tip radius. Calculating $s$ for the case of ice is not yet possible, however, since it would require a full three-dimensional treatment of the dendrite problem including kinetics, which as yet does not exist. It is sufficient here to simply assume that $s$ is a constant to be determined from experiment.

Combining the solvability parameter with the Ivantsov solution then gives a tip radius that is independent of $\sigma_{\infty}$,

$$
R=\frac{B}{\alpha s} R_{\text {kin }}
$$

and a dendrite tip growth velocity

$$
v=\frac{2 D \alpha s}{R_{\text {kin }} B^{2}} \frac{c_{\text {sat }}}{c_{\text {solid }}} \sigma_{\infty},
$$

which depends linearly on $\sigma_{\infty}$.

Experiments with ice dendrites growing in air have found that $v$ is indeed approximately linearly dependent on $\sigma_{\infty}$, as expected (while $R$ is too small to be measured optically with good accuracy). For dendrites at $T=-15^{\circ} \mathrm{C}$ growing in air at a pressure of $1 \mathrm{~atm}$, we have found $v \approx 5 \sigma_{\infty} \mu \mathrm{m} \mathrm{s}^{-1}$, which indicates $R \approx 1.2 \mu \mathrm{m}$ and $\alpha s \approx 0.25$. At $-5^{\circ} \mathrm{C}$ we have $v \approx 10 \sigma_{\infty} \mu \mathrm{m} \mathrm{s}^{-1}$, giving $R \approx 1.5 \mu \mathrm{m}$ and $\alpha s \approx 0.2$. The lack of faceting near fast-growing dendrite tips further suggests that $\alpha \approx 1$. Experimental data are shown above in figure 10 .

\subsection{Full modelling of dendrite growth}

There exists an extensive literature on modelling dendrite growth in numerous different physical situations to varying levels of approximation, which I will not cover here. Results from analytic calculations reveal that a stable dendrite tip, with time-independent $v_{\text {tip }}$ and $R_{\text {tip }}$, is only possible when the capillary and/or kinetic perturbations include some spatial anisotropy. For a purely isotropic material, available for example in computer simulations, the tip frequently splits as 
the dendritic structure grows. We essentially ignored the anisotropy inherent in $\alpha$ in the above simple discussion, but $\alpha$ is certainly anisotropic and we expect that the degree of anisotropy is important for determining the value of the solvability parameter $s$. Dendrite theory remains an incomplete theory in that one cannot precisely calculate $s$ even with rather simple (yet realistic) physical systems.

There is also a large literature describing computer modelling of diffusion-limited growth and the formation of dendrites, which again I will not cover here. Computer models can be divided into two broad camps-'front-tracking' models, in which one must keep track of the solidification interface explicitly, and 'phase-field' models, in which the solidification front is numerically smoothed and not explicitly tracked [104-106]. The latter technique is more popular, partly for its computational simplicity. Unfortunately, quantitative agreement between full three-dimensional simulations and experiment is not entirely satisfying even for the simplest and most well-understood experimental systems [104].

Faceting greatly complicates the full modelling of dendritic growth, and to date the analytic theory describing faceted dendrites is limited [107], as are computer models [108,109]. Experimentally, faceted dendrites have been studied at some level in ice [97,110] and as well as in other materials [111]. At a qualitative level our understanding of faceted systems is fairly good, but a full quantitative theory does not exist even for the simplest faceted dendritic growth.

In summary, we find that the relatively simple dendrite theory described above provides a reasonable semi-quantitative description of the growth of the tips of ice dendrites. It gives reasonable agreement with experiment and shows how the growth scales with various parameters. Solving the full problem, including faceting, side-branching, and other factors, remains a formidable task.

\subsection{Electrically and chemically modified ice dendrite growth}

When an electrical potential is applied to a growing ice dendrite, the diffusion equation becomes modified by additional forces, especially near the dendrite tip. The resulting electrically enhanced growth exhibits some interesting behaviour that can be described with an extension of the theoretical treatment above [97]. The normal diffusion equation is replaced by the Smoluchowski equation $[103,112]$, which describes diffusion in the presence of an external force. We then have

$$
\frac{\partial c}{\partial t}=D \vec{\nabla} \cdot(\vec{\nabla} c+c \vec{\nabla} \Phi)
$$

where the external force felt by the water molecules is described as the gradient of the effective potential

$$
\Phi=-\frac{\xi}{k T}(\vec{E} \cdot \vec{E})
$$

where $\xi$ is the molecular polarizability and the electric field is the gradient of the electrical potential $\vec{E}=-\vec{\nabla} \varphi[103]$. Again we can assume the slow-growth limit and take $\partial c / \partial t \approx 0$. The continuity equation at the interface yields the normal component of the surface growth rate as

$$
v_{\mathrm{n}}=\left.\frac{D}{c_{\text {solid }}} \hat{n} \cdot(\vec{\nabla} c+c \vec{\nabla} \Phi)\right|_{\text {surf }} .
$$

The boundary condition at the crystal surface is now changed by the fact that the applied potential changes the equilibrium vapour pressure, which we can see most easily in the spherical 
case. Consider a spherical droplet of radius $R$ and charge $Q$, where we assume the charge is localized at the droplet surface. The electrostatic self-energy of this charge

$$
U_{\text {electrostatic }}=\frac{1}{2} \frac{1}{4 \pi \varepsilon_{0}} \frac{Q^{2}}{R}
$$

is independent of the dielectric constant of the droplet, since there is zero field below the surface. If we pull one neutral molecule out of the droplet, then this changes $U_{\text {electrostatic }}$ by the amount

$$
\begin{aligned}
\mathrm{d} U_{\text {electrostatic }} & =-\frac{1}{8 \pi \varepsilon_{0}} \frac{Q^{2}}{R^{2}} \frac{\partial R}{\partial N} \\
& =-\frac{1}{32 \pi^{2} \varepsilon_{0}} \frac{Q^{2}}{c_{\text {solid }} R^{4}} .
\end{aligned}
$$

If the molecule has a nonzero polarizability, then pulling the molecule off the droplet releases the polarization energy

$$
\begin{aligned}
\mathrm{d} U_{\text {polar }} & =\xi E^{2} \\
& =\xi \frac{1}{16 \pi^{2} \varepsilon_{0}^{2}} \frac{Q^{2}}{R^{4}},
\end{aligned}
$$

where $\xi \approx 3.4 \times 10^{-39} \mathrm{C}^{2} \mathrm{~m} \mathrm{~N}^{-1}$ for a water molecule.

These energies contribute to the equilibrium vapour pressure like $\mathrm{e}^{\mathrm{d} U / k T}$, yielding for the spherical case (ignoring the surface energy contribution)

$$
c_{\text {surface }} \approx c_{\text {sat }}\left(1+\frac{v_{\mathrm{n}}}{\alpha v_{\text {kin }}}-\frac{R_{\mathrm{es}}^{2}}{R^{2}}+\frac{R_{\mathrm{pol}}^{2}}{R^{2}}\right),
$$

where $R_{\mathrm{es}}^{2}=\varepsilon_{0} \varphi_{0}^{2} / 2 c_{\text {solid }} k T, R_{\text {pol }}^{2}=\xi \varphi_{0}^{2} / k T$, and $\varphi_{0}=Q / 4 \pi \varepsilon_{0} R$ is the electrical potential applied to the droplet. Note the presence of both electrostatic and polarization terms in this expression, the latter being zero for nonpolar molecules. In the case of ice $R_{\mathrm{es}} \approx 0.2\left(\varphi_{0} / 1000 \mathrm{~V}\right) \mu \mathrm{m}$ and $R_{\mathrm{pol}} \approx 1.0\left(\varphi_{0} / 1000 \mathrm{~V}\right) \mu \mathrm{m}$.

The modified Ivantsov relation then becomes [97]

$$
v \approx \frac{2 D}{B R} \frac{c_{\mathrm{sat}}}{c_{\mathrm{solid}}}\left[\sigma_{\infty}-\frac{R_{\mathrm{kin}}^{*}}{R}+C \frac{R_{\mathrm{elec}}^{2}}{R^{2}}\right]
$$

with

$$
R_{\text {elec }}^{2}=\frac{2 \varepsilon_{0}}{c_{\text {solid }}} \frac{\varphi_{0}^{2}}{B^{2} k T},
$$

where $C=1+2 \Gamma \xi c_{\text {solid }} \sigma_{\infty} / \varepsilon_{0}$ and $\Gamma \approx 0.2$ is a dimensionless geometrical constant. Of course this is only an approximate solution to the Smoluchowski equation, which takes the form of the normal Ivantsov solution (in the slow-growth limit) modified by several small additional terms. We expect that a full numerical solution would yield the same basic result, although probably with additional small geometrical corrections.

In the case of ice $R_{\text {elec }} \approx 40\left(\varphi_{0} / 1000 \mathrm{~V}\right) \mathrm{nm}$ and $2 \xi c_{\text {solid }} / \varepsilon_{0}=23.5$, giving $C \approx 1+4.7 \sigma_{\infty}$ at $T=-5^{\circ} \mathrm{C}$. The electrical perturbation can be incorporated into the solvability relation by defining the modified length $R_{\text {comb }} \equiv R_{\text {kin }}^{*}-C R_{\text {elec }}^{2} / R$ and again assuming that this is the additional length scale necessary for uniquely determining the tip radius. This then yields the new solvability relation

$$
s \approx \frac{2 R_{\mathrm{kin}}^{*} D}{v R^{2}} \frac{c_{\mathrm{sat}}}{c_{\mathrm{solid}}}\left(1-C \frac{R_{\mathrm{elec}}^{2}}{R_{\mathrm{kin}}^{*} R}\right) .
$$


Combining this with the modified Ivantsov relation then yields a quadratic equation for the tip radius [97]

$$
R^{2}-R_{0} R+\frac{C R_{0}}{R_{\text {kin }}^{*}} R_{\text {elec }}^{2}=0,
$$

where $R_{0}$ is the tip radius when $R_{\text {elec }}=0$.

For small values of $R_{\text {elec }}$ the solution of this quadratic equation shows that $R$ decreases, and thus $v$ increases, with increasing applied potential. The quadratic equation has no real roots when $R_{\text {elec }}>\left(R_{\mathrm{kin}}^{*} R_{0} / 4 C\right)^{1 / 2}$, indicating that the above modified dendrite theory cannot be used for large $R_{\text {elec }}$, or equivalently when $\varphi_{0}$ is above some threshold potential $\varphi_{\text {thresh }}$. The lack of real roots indicates that above $\varphi_{\text {thresh }}$ neither surface tension nor attachment kinetics can stabilize the tip radius, at least not in the usual sense. At this point the tip experiences runaway growth as $R \rightarrow 0$ under the influence of the Mullins-Sekerka instability (here enhanced by electric forces). The tip velocity thus increases until it is stabilized by some other mechanism [97].

Electrical effects in the growth of ice crystals from the vapour were first described by Mason and collaborators [113,114], and were subsequently quantified as a function of the applied electrical potential [97, 103, 115-117]. The existence of a threshold potential was established in these measurements, and the tip velocity as a function of $\varphi_{0}$ below threshold was found to be in good semi-quantitative agreement with the above theory. Observations measuring the dendrite tip velocities as a function of supersaturation $\sigma_{\infty}$ as well as $\varphi_{0}$ revealed a number of features expected from the theory [97]. The observations showed a threshold potential of $\varphi_{0} \approx 1000 \mathrm{~V}$, which is roughly independent of $\sigma_{\infty}$, consistent with the above theoretical expectations.

Interestingly, trace chemical additives in the air can have a dramatic effect on electric needle growth, even when the concentration level is too low to produce any perceptible change in normal crystal growth. In particular, chemical additives can induce the electric needle growth to be along the ice $c$-axis, and such needles grow approximately three times faster than electric needles in clean air, as is shown in figure 10. A number of chemical additives at the $100 \mathrm{ppm}$ level produce these rapidly growing needles, including various alcohols and acids, as well as chloroform, xylenes, methylene chloride, and even gasoline vapour [97]. Acetic acid is particularly effective even at concentrations as low as $1 \mathrm{ppm}$, which produces essentially no change in the normal crystal growth. It appears likely that the additives are concentrated by the electric fields and gradients near the needle tip, thereby enhancing the chemical effects.

The observations indicate that growing needles have tip radii of $R^{*} \approx 360 \mathrm{~nm}$ for clean air electric needles at $T=-5^{\circ} \mathrm{C}$, as determined using the Ivantsov relation, again roughly independent of $\sigma_{\infty}$. In the presence of chemical additives the higher electric needle velocities imply $R^{*} \approx 90 \mathrm{~nm}$ at $\varphi_{0}=2000 \mathrm{~V}$, also roughly independent of $\sigma_{\infty}$. The tip stabilization method at high applied potentials is still not understood.

\section{Outlook}

A close look at the formation of an individual snow crystal reveals that this is a remarkably rich and complex event. Although it involves little more than the organization of water molecules into a crystalline lattice, many questions about the growth process remain unanswered.

Dendritic structuring and pattern formation-both ubiquitous results of diffusion-limited growth-are one fascinating part of the snow crystal story. Dendrite theory provides a satisfactory semi-quantitative picture of this phenomenon, at least for the growth of welldeveloped dendrites with near-constant tip radii and tip growth velocities. The theory does 
not include faceting at present, as this has been found to be a formidable problem. Progress toward a full theory of dendrite growth, including attachment kinetics, has been slow for many years and is likely to continue so.

However, there are certainly several interesting areas in dendritic snow crystal growth that remain unexplored. Can one better understand the transition from faceted growth to dendritic growth in a quantitative way? What happens in different background gases, and at different pressures? Can one further understand the effects of external stimuli on dendrite growth, such as electrical and chemical effects? I suspect experiment will lead the way in these areas, especially quantitative experiments which measure growth under well-controlled conditions. It seems likely to me that further surprises await the experimenter who ventures to explore these and other questions about ice dendrite growth.

The growth of plain hexagonal ice prisms remains perhaps the most puzzling aspect of snow crystal growth, in spite of its apparent simplicity. The snow crystal morphology diagram, well documented now for 75 years, has still not been explained at even a qualitative level. Surface melting appears to play an important role, as do unusual growth instabilities, but major problems remain unsolved. The morphology diagram is continually demonstrated to us by snow crystals falling from the sky in copious numbers. Surely we can understand why snow crystal morphology has this unusual dependence on temperature. In my opinion, this area is ripe for progress on both the experimental and theoretical fronts.

On the experimental side, we still do not possess accurate measurements of ice growth as a function of temperature and supersaturation for the two principal facets, which would tell us much about $\alpha\left(T, \sigma_{\text {surf }}\right)$ and the growth mechanisms. This situation will likely change soon as new experiments address the systematic problems that have plagued earlier efforts. Not only do we need measurements of prisms growing on substrates in near-vacuum, but also measurements of levitated prisms growing in various background gas pressures. The combination of data will speak volumes about the different instabilities that appear to dominate snow crystal growth under different conditions.

On the theoretical side, we need a model of how crystals grow in the presence of surface melting. This implies a better understanding of crystal growth in the near-equilibrium limit, when evaporation and growth are simultaneously occurring at rates that far exceed the net growth rate. We also need to broaden our horizons when it comes to crystal growth instabilities, including those relating to the growth of thin ice plates and needles. This is an area of considerable general interest, as it appears that instabilities of many varieties abound in growing systems.

There is also the vast, unexplored topic concerning the effects of chemical impurities on ice growth. I suspect many surprises are in store when we begin to understand the chemical catalysis of crystal growth in general, and of ice growth in particular. Again it is likely that quantitative experiments will lead the way in exploring this potentially exciting territory.

Snow crystal formation provides a fascinating case study of the physics of crystal growth, requiring a deep understanding of the nanoscale dynamics of solidification and pattern formation. By studying snow crystals, one can gain insights into many fundamental aspects of materials science. There is much that remains to be learned.

\section{References}

[1] Dash J G and Wettlaufer J S 2003 The surface physics of ice in thunderstorms Can. J. Phys. 81 201-7

[2] Baker M B and Dash J G 1994 Mechanism of charge transfer between colliding ice particles in thunderstorms J. Geophys. Res. 99 10621-6

[3] Black R A and Hallett J 1998 The mystery of cloud electrification Am. Sci. 86 526-34 
[4] Clary D C 1996 Molecules on ice Science 2711509

[5] Zondlo M A, Barone S B and Tolbert M A 1998 Condensed-phase products in heterogeneous reactions: $\mathrm{N}_{2} \mathrm{O}_{5}$, $\mathrm{ClONO}_{2}$, and $\mathrm{HNO}_{3}$ reacting on ice films at $185 \mathrm{~K}$ J. Phys. Chem. A $1025735-48$

[6] Debenedetti P G and Stanley H E 2003 Supercooled and glassy water Phys. Today 56 40-6

[7] Frank F C 1982 Snow crystals Contemp. Phys. 23 3-22

[8] Mason B J 1992 Snow crystals, natural and man made Contemp. Phys. 33 227-43

[9] Nakaya U 1954 Snow Crystals: Natural and Artificial (Cambridge: Harvard University Press)

[10] Kobayashi T and Kuroda T 1987 Snow crystals Morphology of Crystals—Part B ed I Sunagawa (Tokyo: Terra Scientific) pp 645-743

[11] Wang P K 2002 Shape and microdynamics of ice particles and their effects in cirrus clouds Adv. Geophys. 45 $1-258$

[12] Kepler J 1966 On the Six-Cornered Snowflake (Oxford: Clarendon) (Engl. Transl.)

[13] Scoresby W 1820 An Account of the Arctic Regions with a History and Description of the Northern WhaleFishery (Archibald Constable)

[14] Chickering F 1864 Cloud Crystals: A Snow Flake Album (New York: Appleton Company)

[15] Bentley W A and Humphreys W J 1931 Snow Crystals (New York: McGraw-Hill)

[16] Libbrecht K 2004 The Little Book of Snowflakes (Stillwater, MN: Voyageur)

[17] Kobayashi T 1966 On the variation of ice crystal habit with temperature Conf. on Physics of Snow and Ice (Sapporo, Japan) pp 95-104

[18] Magono C and Lee C W 1966 Meteorological classification of natural snow crystals J. Fac. Sci. Hokkaido Univ.

[19] Libbrecht K 2003 The Snowflake: Winter's Secret Beauty (Stillwater, MN: Voyageur)

[20] Kobayashi T 1961 The growth of snow crystals at low supersaturations Phil. Mag. 6 1363-70

[21] Bailey $\mathrm{M}$ and Hallett $\mathrm{J} 2004$ Growth rates and habits of ice crystals between $-20^{\circ} \mathrm{C}$ and $-70^{\circ} \mathrm{C} \mathrm{J}$. Atmos. Sci. $61514-44$

[22] Hallett J and Mason B J 1958 The influence of temperature and supersaturation on the habit of ice crystals grown from the vapour Proc. R. Soc. Lond. A 247 440-53

[23] Keller V W and Hallett J 1982 Influence of air velocity on the habit of ice crystal growth from the vapor $J$. Cryst. Growth 60 91-106

[24] Hallett J 1984 How snow crystals grow Am. Sci. 72 582-9

[25] Yamashita A 1974 Studies on the ice crystals using a large cloud chamber Kisho Kenkyu Noto, Meteor. Soc. Japan 123 47-94 (in Japanese)

[26] Libbrecht K G and Yu H 2001 Crystal growth in the presence of surface melting: supersaturation dependence of the growth of columnar crystals J. Cryst. Growth 222 822-31

[27] Libbrecht K G 2003 Explaining the growth of thin ice crystal plates with structure-dependent attachment kinetics J. Cryst. Growth 258 168-75

[28] Markov I V 1995 Crystal Growth for Beginners: Fundamentals of Nucleation, Crystal Growth, and Epitaxy (Singapore: World Scientific)

[29] Shibkov A A et al 2003 Morphology diagram of nonequilbrium patterns of ice crystals growing in supercooled water Physica A 319 65-79

[30] Mason B J 1971 The Physics of Clouds (Oxford: Clarendon)

[31] Nelson J 2001 Growth mechanisms to explain the primary and secondary habits of snow crystals Phil. Mag. A $812337-73$

[32] Huang J and Bartell L S 1995 Kinetics of homogeneous nucleation in the freezing of large water clusters $J$. Phys. Chem. 99 3924-31

[33] Petrenko V F and Whitworth R W 1999 Physics of Ice (Oxford: Oxford University Press)

[34] Saito Y 1996 Statistical Physics of Crystal Growth (Singapore: World Scientific)

[35] Hirth J P and Pound G M 1963 Condensation and Evaporation (Oxford: Pergamon)

[36] Liu S J, Huang H C and Woo C H 2002 Schwoebel-Ehrlich barrier: from two to three dimensions Appl. Phys. Lett. 80 3295-7

[37] Gonda T and Yamazaki T 1978 Morphology of ice droxtals grown from supercooled water droplets J. Cryst. Growth 45 66-9

[38] Elbaum M 1991 Roughening transition observed on the prism facet of ice Phys. Rev. Lett. 67 $2982-5$

[39] Libbrecht K G 2003 Growth rates of the principle facets of ice between $-10^{\circ} \mathrm{C}$ and $-40^{\circ} \mathrm{C} \mathrm{J}$. Cryst. Growth 247 530-40

[40] Wood S E, Baker M B and Calhoun D 2001 New model for the vapor growth of hexagonal ice crystals in the atmosphere J. Geophys. Res. 106 4845-70 
[41] Gonda T and Komabayasi M 1971 Skeletal and dendritic structures of ice crystal as a function of thermal conductivity and vapor diffusivity J. Meteor. Soc. Japan 49 32-41

[42] Libbrecht K G 1999 Cylindrically symmetric Green's function approach for modeling the crystal growth morphology of ice Phys. Rev. E $601967-73$

[43] Yokoyama E and Kuroda T 1990 Pattern formation in growth of snow crystals occurring in the surface kinetic process and the diffusion process Phys. Rev. A 41 2038-49

[44] Yokoyama E 1993 Formation of patterns during growth of snow crystals J. Cryst. Growth 128 251-7

[45] Terra Analysis 1998 Quickfield finite-element analysis system

[46] Eames I W, Marr N J and Sabir H 1997 The evaporation coefficient of water: a review Int. J. Heat Mass Transfer 40 2963-73

[47] Ohara M and Reed R C 1973 Modeling Crystal Growth Rates from Solution (Englewood Cliffs, NJ: PrenticeHall)

[48] Chernov A A 1993 Roughening and melting of crystalline surfaces Prog. Cryst. Growth Charact. 26 195-218

[49] Conrad E H 1992 Surface roughening, melting, and faceting Prog. Surf. Sci. 39 65-116

[50] van Veenendall E et al 1999 Kinetic roughening of the kossel (1 0 0 $)$ ) surface J. Cryst. Growth 198 22-6

[51] Dash J G, Fu H and Wettlaufer J S 1995 The premelting of ice and its environmental consequences Rep. Prog. Phys. 58 115-67

[52] Dash J G 1989 Surface melting Contemp. Phys. 30 89-100

[53] Lindemann F A 1910 Z. Phys. 11609

[54] Grimvall G and Sjodin S 1974 Correlation of properties of materials to Debye and melting temperatures Phys. Scr. 10 340-52

[55] Martin C J and O'Connor D A 1977 An experimental test of Lindemann's melting law J. Phys. C: Solid State Phys. $103521-6$

[56] Schmidt M et al 1997 Experimental determination of the melting point and heat capacity for a free cluster of 139 sodium atoms Phys. Rev. Lett. 79 99-102

[57] Lacmann R and Stranski I N 1972 The growth of snow crystals J. Cryst. Growth 13/14 236-40

[58] Doppenschmidt A and Butt H-J 2000 Measuring the thickness of the liquid-like layer on ice surfaces with atomic force microscopy Langmuir 16 6709-14

[59] Dosch H, Lied A and Bilgram J H 1995 Glancing-angle x-ray scattering studies of the premelting of ice surfaces Surf. Sci. 327 145-64

[60] Wei X et al 2002 Sum-frequency spectroscopic studies of ice interfaces Phys. Rev. B 66085401

[61] Bolton K and Pettersson J B C 2000 A molecular dynamics study of the long-time ice Ih surface dynamics J. Phys. Chem. B 104 1590-5

[62] Furukawa Y and Nada H 1997 Anisotropic surface melting of an ice crystal and its relationship to growth forms J. Phys. Chem. B 101 6167-70

[63] Kroes G-J 1992 Surface melting of the (0001) face of tip4p ice Surf. Sci. 275 365-82

[64] Bienfait M 1992 Roughening and surface melting transitions: consequences on crystal growth Surf. Sci. 272 1-9

[65] Kuroda T and Lacmann R 1982 Growth kinetics of ice from the vapour phase and its growth forms J. Cryst. Growth 56 189-205

[66] Kandel D and Kaxiras E 2000 The surfactant effect in semiconductor thin-film growth Solid State Phys. 54 219-62

[67] Muller B H, Schmidt Th and Henzler M 1997 Growth and melting of a Pb monolayer on Cu(111) Surf. Sci. 376 123-32

[68] Nelson J and Knight C A 1996 A new technique for growing crystals from the vapor J. Cryst. Growth 169 795-7

[69] Gonda T 1976 The growth of small ice crystals in gases of high and low pressures J. Meteor. Soc. Japan $\mathbf{5 4}$ 233-40

[70] Schaefer V J 1952 Continuous cloud chamber for studying small particles in the atmosphere Indust. Eng. Chem. 44 1381-3

[71] Mason B J 1961 The growth of snow crystals Sci. Am. 204 120-31

[72] Bryant G W, Hallett J and Mason B J 1959 The epitaxial growth of ice on single-crystalline substrates J. Phys. Chem. Solids 12 189-95

[73] Beckmann W, Lacmann R and Blerfreund A 1983 Growth rates and habits of crystals grown from the vapor phase J. Phys. Chem. 87 4142-6

[74] Foster T C and Hallett J 1993 Ice crystals produced by expansion: experiments and application to aircraftproduced ice J. Appl. Meteor. 32 716-28 
[75] Anderson B J and Hallett J 1976 Supersaturation and time dependence of ice nucleation from the vapor on single crystal substrates J. Atmos. Sci. 33 822-32

[76] Zepeda S, Yeh Y and Orme C A 2001 Atomic force microscope chamber for in situ studies of ice Rev. Sci. Instrum. 72 4159-63

[77] Bryant G W and Mason B J 1960 Photolytic de-activation of silver iodide as an ice-forming nucleus $Q . J . R$ Meteor. Soc. 86 354-7

[78] Anderson B J and Hallett J 1979 Influence of environmental saturation and electric field on growth and evaporation of epitaxial ice crystals J. Cryst. Growth $\mathbf{4 6} 427-44$

[79] Rukuta N and Takahashi T 1999 The growth of atmospheric ice crystals: a summary of findings in vertical supercooled cloud tunnel studies J. Atmos. Sci. 56 1963-79

[80] Swanson B D, Bacon N J, Davis E J and Baker M B 1999 Electrodynamic trapping and manipulation of ice crystals Q. J. R. Meteor. Soc. 125 1039-58

[81] Furukawa Y and Kohata S 1993 Temperature dependence of the growth form of negative crystal in an ice single crystal and evaporation kinetics for its surfaces J. Cryst. Growth 129 571-81

[82] Nenow D, Trayanov A and Pavlovska A 1982 Surface roughness and kinetics of spontaneous transformation of negative crystals Cryst. Res. Technol. 17 1179-85

[83] Libbrecht K G 2004 A critical look at ice crystal growth data Preprint cond-mat/0411662

[84] Beckmann W and Lacmann R 1982 Interface kinetics of the growth and evaporation of ice single crystals from the vapour phase-II. Measurements in a pure water vapour environment J. Cryst. Growth 58 $433-42$

[85] Nelson J and Knight C 1998 Snow crystal habit changes explained by layer nucleation J. Atmos. Sci. 55 1452-65

[86] Cho N and Hallett J 1984 Epitaxial ice crystal growth on covellite (Cus) - I. Influence of misfit strain on the growth of non-thickening crystals J. Cryst. Growth 69 317-24

[87] Beckmann W 1982 Interface kinetics of the growth and evaporation of ice single crystals from the vapour phase-III. Measurements under partial pressures of nitrogen J. Cryst. Growth 58 443-51

[88] Mason B J, Bryant G W and van den Heuval A P 1963 Growth habits and surface structure of ice crystals Phil. Mag. 8 505-9

[89] van den Heuval A P and Mason B J 1959 Habit of ice crystals grown in hydrogen, carbon dioxide and air at reduced pressure Nature 184 519-20

[90] Libbrecht K G 2004 unpublished

[91] Kikuchi K and Hogan A W 1979 Properties of diamond dust type ice crystals observed in summer season at Amundsen-Scott South Pole Station, Antarctica J. Meteor. Soc. Japan 57 180-90

[92] Shimada W and Furukawa Y 1997 Pattern formation of ice crystals during free growth in supercooled water J. Phys. Chem. B 101 6171-3

[93] Maruyama M, Nishida T and Sawada T 1997 Crystal shape of high-pressure ice Ih in water and roughening transition of the (1010) plane J. Phys. Chem. B 101 6151-3

[94] Maruyama M, Ashida T and Knight C A 1999 Disk crystals of ice grown in air-free water: no effect of dissolved air on the morphology J. Cryst. Growth 205 391-4

[95] Ben-Jacob E 1993 From snowflake formation to growth of bacterial colonies-I. Diffusive patterning in azoic systems Contemp. Phys. 34 247-73

[96] Ben-Jacob E 1997 From snowflake formation to growth of bacterial colonies-II. Cooperative formation of complex colonial patterns Contemp. Phys. 38 205-41

[97] Libbrecht K G, Crosby T and Swanson M 2002 Electrically enhanced free dendrite growth in polar and nonpolar systems J. Cryst. Growth $240241-54$

[98] Langer J S 1980 Instabilities and pattern formation in crystal growth Rev. Mod. Phys. 52 1-28

[99] Mullins W W and Sekerka R F 1964 Stability of a planar interface during solidification of a dilute binary alloy J. Appl. Phys. 35 444-51

[100] Kessler D A, Koplik J and Levine H 1988 Pattern selection in fingered growth phenomena Adv. Phys. 37 255-339

[101] Brener E A and Mel'nikov V I 1991 Pattern selection in two-dimensional dendritic growth Adv. Phys. 40 53-97

[102] Brener E A 1996 Three-dimensional dendritic growth J. Cryst. Growth 166 339-46

[103] Libbrecht K G and Tanusheva V M 1999 Cloud chambers and crystal growth: effects of electrically enhanced diffusion on dendrite formation from neutral molecules Phys. Rev. E 59 3253-61

[104] Karma A and Rappel W J 1997 Phase-field simulation of three-dimensional dendrites: Is microscopic solvability theory correct? J. Cryst. Growth 174 54-64

[105] Wang S L et al 1993 Thermodynamically consistent phase-field models for solidification Physica D 69 189-200

[106] Wheeler A A, Murray B T and Schaefer R J 1993 Computation of dendrites using a phase field model Physica D 66 243-62

[107] Bedia M A and Amar M B 1995 Faceting in free dendrite growth Phys. Rev. E 51 1268-75 
[108] Uehara T and Sekerka R F 2003 Phase field simulations of faceted growth for strong anisotropy of kinetic coefficient J. Cryst. Growth 254 251-61

[109] Taylor J E and Cahn J W 1998 Diffuse interfaces with sharp corners and facets: phase field models with strongly anisotropic surfaces Physica D 112 381-411

[110] Gonda T and Nakahara S 1997 Dendritic ice crystals with faceted tip growing from the vapor phase $J$. Cryst. Growth 173 189-93

[111] Maurer J et al 1989 Faceted dendrites in the growth of $\mathrm{NH}_{4} \mathrm{Br}$ crystals Europhys. Lett. 8 67-72

[112] Chandrasekhar S 1943 Rev. Mod. Phys. 1515

[113] Bartlett J T, van den Heuval A P and Mason B J 1963 The growth of ice crystals in an electric field Z. Angew. Math. Phys. 14 599-610

[114] Evans L F 1973 The growth and fragmentation of ice crystals in an electric field J. Atmos. Sci. 30 1657-64

[115] Libbrecht K G 1998 Electrically induced morphological instabilities in free dendrite growth Phys. Rev. Lett. 81 176-9

[116] Brener E A 1999 Comment on electrically induced morphological instabilities in free dendrite growth Phys. Rev. Lett. 831698

[117] Libbrecht K G 1999 Comment on electrically induced morphological instabilities in free dendrite growthLibbrecht and Tanusheva reply Phys. Rev. Lett. 831699 